\title{
A causal inference approach to measure the vulnerability of urban metro systems
}

\author{
Nan Zhang ${ }^{1} \cdot$ Daniel J. Graham $^{1}$ (D) . Daniel Hörcher ${ }^{1} \cdot$ Prateek Bansal $^{1}$ \\ Published online: 26 January 2021 \\ (c) The Author(s) 2021
}

\begin{abstract}
Transit operators need vulnerability measures to understand the level of service degradation under disruptions. This paper contributes to the literature with a novel causal inference approach for estimating station-level vulnerability in metro systems. The empirical analysis is based on large-scale data on historical incidents and population-level passenger demand. This analysis thus obviates the need for assumptions made by previous studies on human behaviour and disruption scenarios. We develop four empirical vulnerability metrics based on the causal impact of disruptions on travel demand, average travel speed and passenger flow distribution. Specifically, the proposed metrics based on the irregularity in passenger flow distribution extends the scope of vulnerability measurement to the entire trip distribution, instead of just analysing the disruption impact on the entry or exit demand (that is, moments of the trip distribution). The unbiased estimates of disruption impact are obtained by adopting a propensity score matching method, which adjusts for the confounding biases caused by non-random occurrence of disruptions. An application of the proposed framework to the London Underground indicates that the vulnerability of a metro station depends on the location, topology, and other characteristics. We find that, in 2013, central London stations are more vulnerable in terms of travel demand loss. However, the loss of average travel speed and irregularity in relative passenger flows reveal that passengers from outer London stations suffer from longer individual delays due to lack of alternative routes.
\end{abstract}

Keywords Vulnerability · Urban metro system - Causal inference $\cdot$ Propensity score matching

Daniel J. Graham

d.j.graham@imperial.ac.uk

Nan Zhang

nan.zhang16@imperial.ac.uk

Daniel Hörcher

d.horcher@imperial.ac.uk

Prateek Bansal

prateek.bansal@imperial.ac.uk

1 Transport Strategy Centre, Department of Civil and Environmental Engineering, Imperial College London, London, UK 


\section{Introduction}

Metros, also known as subways or rapid transit, have become a vital component of public transport. With the advantage of large capacity and high-frequency services, 178 metro systems worldwide carried a total of 53,768 million trips in 2017 (International Union of Public Transport 2018). Incidents occur frequently in urban metro systems, mainly due to supply-side failures (e.g., signal failures), sudden increase in travel demand (e.g., public concert or football matches) and change in weather conditions (Brazil et al. 2017; Melo et al. 2011; Wan et al. 2015). These incidents can cause service delays and overcrowding, which in turn lead to safety concerns and potential losses in social welfare. For instance, the London Underground encountered 7973 service disrupting incidents of above 2 min duration between April 2016 and April 2017, causing a total loss of around 34 million customer hours (Transport for London 2017, 2019). The Singapore Mass Rapid Transit experienced 47 severe delays that lasted over 30 min between 2015 and 2017 (Land Transport Authority 2017).

Operators may consider investing in new technologies to improve metro facilities and mitigate the effect of incidents. For instance, the New York City Subway was in a state of emergency in June 2017 after a series of derailments, track fires and overcrowding incidents. The Metropolitan Transportation Authority invested over $\$ 8$ billion to stabilise and modernise the incident-plagued metro system (Metropolitan Transportation Authority 2019). It is apparent that metros are willing to invest in their infrastructure systems, but it is often not known how those investments compare in achieving improvements. To facilitate project selection, metros are increasingly relying on disaggregate performance metrics that reveal the most vulnerable parts of the network. Performance can be measured in various ways. Popular examples are risk, resilience, reliability and vulnerability related metrics. These concepts are often confused by researchers as well as well as practitioners. Interested readers can refer to Faturechi and Miller-Hooks (2015) and Reggiani et al. (2015) to understand the most agreed relationship among these concepts. In this paper, we focus on the vulnerability of urban metro systems, where the performance measures of interest are passenger demand, average travel speed and passenger flow distribution.

Since the 1990 s, the concept of vulnerability has been widely used to characterise the performance of transport systems (Mattsson and Jenelius 2015; Reggiani et al. 2015), which is often defined as a measure of susceptibility of the transport system to incidents (Berdica 2002; Jenelius et al. 2006; O'Kelly 2015). In this study, the vulnerability of metro systems refers to the extent of degradation in the level of service due to service disruptions. Service disruptions are defined as events that interrupt normal train operations for a specific period of time. ${ }^{1}$ Disruptions should be distinguished from the broader term "incidents", as incidents might not always affect services. Examples of such incidents include elevator failure or corridor congestion in metro stations. Vulnerability metrics can measure the consequences of service interruptions, in the form of performance outputs such as train kilometres, passenger volumes or the quality of travelling. For operators, such metrics have important implications in identifying weak stations or links in metro systems and efficiently allocating resources to the most

\footnotetext{
1 Five minutes to ten minutes are commonly used thresholds to define disruptions (Zhang et al. 2016). Different metro systems around the world adopt several thresholds, primarily on the basis of the regular frequency of operations.
} 
affected areas. Given the rising interest in utilising vulnerability metrics in disruption prevention and management, obtaining an accurate measure of such metrics is crucial.

Traditionally, vulnerability in urban metros is investigated based on complex network theory and graph theory. Complex network theory converts metro networks into graphs, which enables the quantitative measurement of vulnerability in metro systems (Chopra et al. 2016; Derrible and Kennedy 2010; Yang et al. 2015). The adoption of graph theory has facilitated the evolution of vulnerability indicators from simply capturing the characteristics of network topology to also considering travel demand patterns and their land use dependencies (Jiang et al. 2018). However, most of these studies rely on simulation-based approaches to quantify vulnerability under hypothetical scenarios of disruptions. These simulation experiments are based on assumptions, both in terms of passenger behaviour and the type and scale of disruptions (Lu 2018; Sun and Guan 2016; Sun et al. 2015, 2018). With an empirical approach, such assumptions can be avoided, and thus more reliable metrics of vulnerability can be achieved using historical evidence.

The empirical approach is rare but not unique in the literature. The exception we are aware of is Sun et al. (2016), who first detect incidents based on abnormal ridership and use the real incidents data to assess the vulnerability of the metro system. However, their method has some limitations. First, they assume the occurrence of incidents to be random, which is a strict and unrealistic assumption as we demonstrate in this study. Also, the abnormal ridership may not be a good indicator of incidents if the fluctuation in ridership are merely manifestations of changes in travel demand due to external factors.

This paper proposes a novel alternative methodology to quantify vulnerability, by empirically estimating the causal impact of service disruptions on travel demand, average travel speed and passenger flow distribution at station-level. The application of a propensity score matching method accounts for the non-randomness of disruptions and ensures unbiasedness of the causal estimates. We make this approach comprehensive for the entire network, including stations where disruptions are not observed, by predicting the level of vulnerability at these stations with a random forest algorithm. In this way, we eliminate the need for ad hoc assumptions on passenger behaviour and the nature of disruptions.

We use London Underground as a case study and apply the methodology with largescale automated fare collection and incident data. The station-level vulnerability is heterogeneous among the network, depending on the considered performance metrics. In terms of the demand loss and gross speed loss (overall delay), the most affected stations are more likely to be found in Central London areas. When considering average speed loss (individual delay) and irregularity in relative passenger flows, the most affected stations are scattered around outer London areas due to lack of alternative routes. These results can potentially aid investment decisions of metro operators.

The rest of paper is organised as follows. Section "Literature review" reviews the literature on vulnerability measurement and disruption impact analysis in urban metro systems. Section 3 presents our empirical framework to compute vulnerability metrics. This section discusses the proposed causal inference approach to estimate the unbiased disruption impact, which is the key input in building vulnerability metrics. In "Case study: London underground" section, we analyse the vulnerability of London Underground as a case study. Results are discussed in "Results and discussions" section. Finally, "Conclusions and future work" section concludes and highlights the potential avenues for future research. 


\section{Literature review}

Below we provide a contextual review of previous studies related to vulnerability measurement. In "Measuring the vulnerability of metro systems" section, we review the literature on vulnerability quantification in rail transit networks, while "Estimating disruption impact" section investigates previous attempts to estimate the impact of disruptions.

\section{Measuring the vulnerability of metro systems}

There are two traditional methods used to build vulnerability indicators of metro systemstopology-based and system-performance-based analysis.

The topological methods rely on complex network theory to convert the metro network into a scale-free graph, in which nodes represent metro stations, edges represent links between directly connected stations and the weight associated with each edge is computed based on travel time or distance (Derrible and Kennedy 2010; Mattsson and Jenelius 2015; Zhang et al. 2011). The changes in the system's connectivity are reflected on graphs by removing nodes or links and vulnerability is entirely governed by the topological structure. For instance, the location importance of metro stations or links is indicated by the number of edges connected to a specific node and the fraction of shortest paths passing through the given node/edge (Sun and Guan 2016; Sun et al. 2018; Yang et al. 2015; Zhang et al. 2018b). Network-level efficiency is indicated by the average of reciprocal shortest path length between any origin-destination (OD) pair. Such global indicators capture the overall reachability as well as the service size of a metro system (Sun et al. 2015; Yang et al. 2015).

System-performance-based analyses not only consider the network topology but also incorporate real data on metro operations (e.g., ridership distribution) into vulnerability measurement (M'cleod et al. 2017; Mattsson and Jenelius 2015). For instance, Sun et al. (2018) use a ridership-based indicator - a sum of flows in edges connected with the given node-to complement the topological measures by integrating passengers' travel preferences. Other studies use passenger delay and demand loss as vulnerability indicators (Adjetey-Bahun et al. 2016; M'cleod et al. 2017; Nian et al. 2019; Rodríguez-Núñez and García-Palomares 2014). Specifically, passenger delay is summarised by changes in the weighted average of travel time between all OD pairs due to disruptions where weights are station-level passenger loads. Jiang et al. (2018) suggest integrating land use characteristics around stations into vulnerability measurement because metro systems interact with the external environment during incidents.

To quantify vulnerability based on the aforementioned indicators of the system's performance, almost all previous studies adopt simulation-based approaches and assume hypothetical disruption scenarios. The simplest disruption scenario involves a single station or link closure, assuming one node or edge in the graph is out of service. This incident affects the topology structure and passengers' route choice and the differences in the corresponding performance indicators under normal and disrupted scenarios are quantified to measure vulnerability (Sun et al. 2015). More complex disruption scenarios include the closure of two or more non-adjacent stations, failure of an entire line, and sequential closure of stations until the network crashes (Adjetey-Bahun et al. 2016; Chopra et al. 2016; Sun and Guan 2016; Zhang et al. 2018a, b). Ye and Kim (2019) also discuss the case of partial station closure. 
Simulation-based studies gained popularity because they do not require incident data and can flexibly control simulation settings to imitate a wider range of possible situations. However, researchers have to make many assumptions to infer passengers' response to virtual disruptions. Without observing passengers' movements during real incidents, the validity of the simulation assumptions is questionable. For example, while quantifying passenger delay indicators, Rodríguez-Núñez and García-Palomares (2014) and AdjeteyBahun et al. (2016) assume that all passengers have the same travel speed and they do not change their destinations under disruptions unless there is no available route. However, in reality, passengers can travel at different speeds, leave the metro system, change their destinations, or reroute during disruptions. As a result, especially for system-based analyses, vulnerability metrics obtained from simulation-based studies may not reflect the true changes in the level of service due to disruptions. There is, therefore, scope to improve vulnerability measurement by empirically estimating the impact of disruptions. The advantage of empirical-based methods is that the aforementioned assumptions are no longer needed, and the estimated impacts of disruptions are more reliable. However, the need for largescale datasets is the main drawback of empirical studies.

\section{Estimating disruption impact}

In an urban rail transit context, early attempts to analyse disruption impact relied on surveys. Rubin et al. (2005) conducted a stated preference survey to understand the psychological and behavioural reactions of travellers to the bombing incident, which happened in London during July 2005. They consider passenger's reduced intention of travelling by the London Underground after the attack as the key indicator. Since stated willingness may not reflect real travel behaviour, Zhu et al. (2017) performed a revealed preference survey to investigate travellers' reactions to transit service disruptions in Washington D.C. Metro. By comparing their actual travel choices before and during the metro shutdown, they find a $20 \%$ reduction in demand. Results from such surveys are usually presented as the percentage change in passengers' preferences for travel modes, departure time, and destinations. Although this information is useful, we still need detailed information about delays or demand losses to quantify true disruption impacts. Furthermore, there are inherent limitations of survey-based studies. For instance, repeated observations of a respondent are difficult to collect for a long period because of constraints associated with cost, manpower, recording accuracy, and privacy protection of respondents (Kusakabe and Asakura 2014). A survey sample also cannot cover all passengers, which may lead to biased estimates of disruption impact if the sample is not representative of the population.

With the wide use of automated fare collection facilities in metro systems, smart card data have become a powerful tool for research related to transit operations and travel behaviour (Pelletier et al. 2011). Compared to survey data, the key advantages of smart card data are cost-effectiveness, continuous long-term recording and accurate travel information for each passenger within the system (Kusakabe and Asakura 2014). Therefore, researchers have started using smart card data to analyse disruption impacts. For instance, Sun et al. (2016) develop a method to identify incidents and conduct trip assignments with/without incidents. They estimate the disruption impact by computing the differences between two assignments in terms of ridership distribution and travel time across all OD pairs. This study does not require extra assumption about passengers' reaction because their actual locations and movements are revealed from smart card data. However, they assume that metro disruptions occur randomly, while in reality, factors such as travel demand, 
signalling type, passenger behaviour, operating years, rolling stock characteristics and weather conditions have a significant influence on the likelihood of metro failures (Brazil et al. 2017; Melo et al. 2011; Wan et al. 2015). This is a particularly important consideration because the impact estimated from direct comparison of performance indicators before and after disruptions will be biased under non-random occurrence of disruptions. Specifically, a few factors affecting the impact of disruptions (e.g., passenger behaviour and weather conditions) may also affect the occurrence of disruptions, leading to confounding bias in pre-post comparison estimates (Imbens and Rubin 2015). Some researchers also adopt prediction-based approaches to quantify disruption impact using smart card data. For instance, Silva et al. (2015) propose a framework to predict the exit ridership and model behaviours of passengers under station closure and line segment closure. In a very recent study, Yap and Cats (2020) apply supervised learning approaches to predict the passenger delay caused by incidents. However, these prediction-based studies also cannot disentangle the causal effect of disruptions and can result into biased estimates due to the existence of confounding factors.

Table 1 shows a comparison of recent vulnerability studies and also illustrates the contribution of this research. We conclude this section with a summary of gaps in the literature that we address to obtain more accurate measures of vulnerability:

1. Previous studies on vulnerability metrics of transit systems are largely based on simulation approaches. These studies do not account for the actual behaviour of passengers under disruptions. Basing analyses on empirical data, rather than simulations, obviates the need for making potentially unrealistic assumptions on passengers' movement.

2. In urban metro systems, disruption occurrences can be non-random. Therefore, empirical studies on quantifying disruption impacts should account for this non-randomness to eliminate confounding biases in estimation.

In this paper, we show that both improvements can be made by adopting causal inference methods and calibrating them using large-scale smart card data and incident data. Specifically, the proposed method allows for the non-random occurrence of disruptions and adjusts for potential bias caused by confounding factors. Subsequently, unbiased empirical estimates of disruption impact are used to accurately compute vulnerability metrics of metro systems.

\section{Methodology}

From a methodological point of view, our empirical approach has three stages: first, we apply a causal inference method to estimate the impact of disruptions on station-level travel demand and travel speed (see Sect. 3.1). Then, in Sect. 3.2 we construct vulnerability metrics based on the disruption impact estimated in the first stage. Finally, the third stage imputes $^{2}$ missing vulnerability metrics for non-disrupted stations using machine learning algorithms. Figure 1 illustrates all steps of the proposed empirical framework.

\footnotetext{
2 In Statistics, "imputation" is the process of replacing missing data with substituted values. Here we retrieve these missing values based on a relationship between vulnerability metrics and covariates of the disrupted stations.
} 


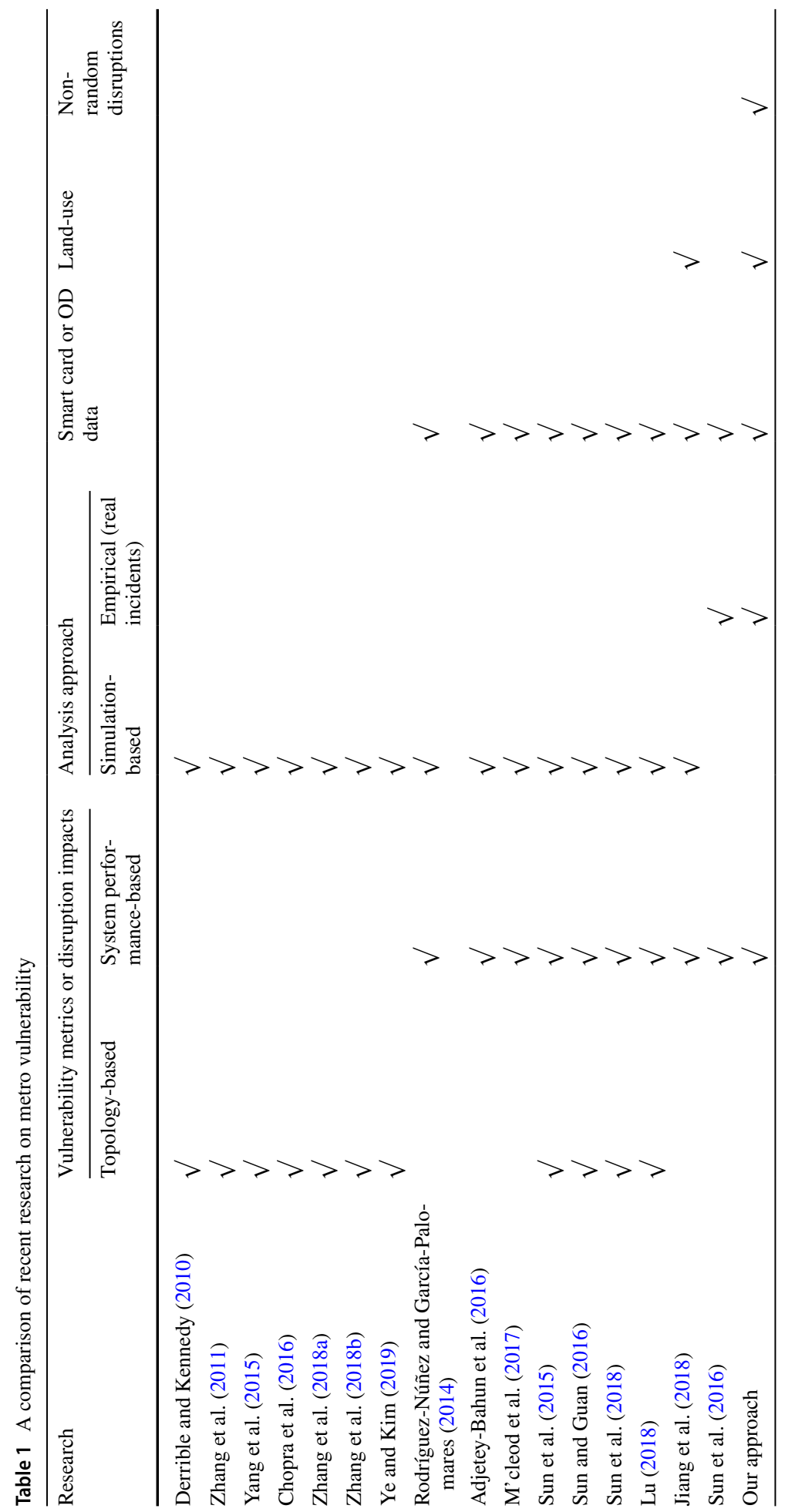




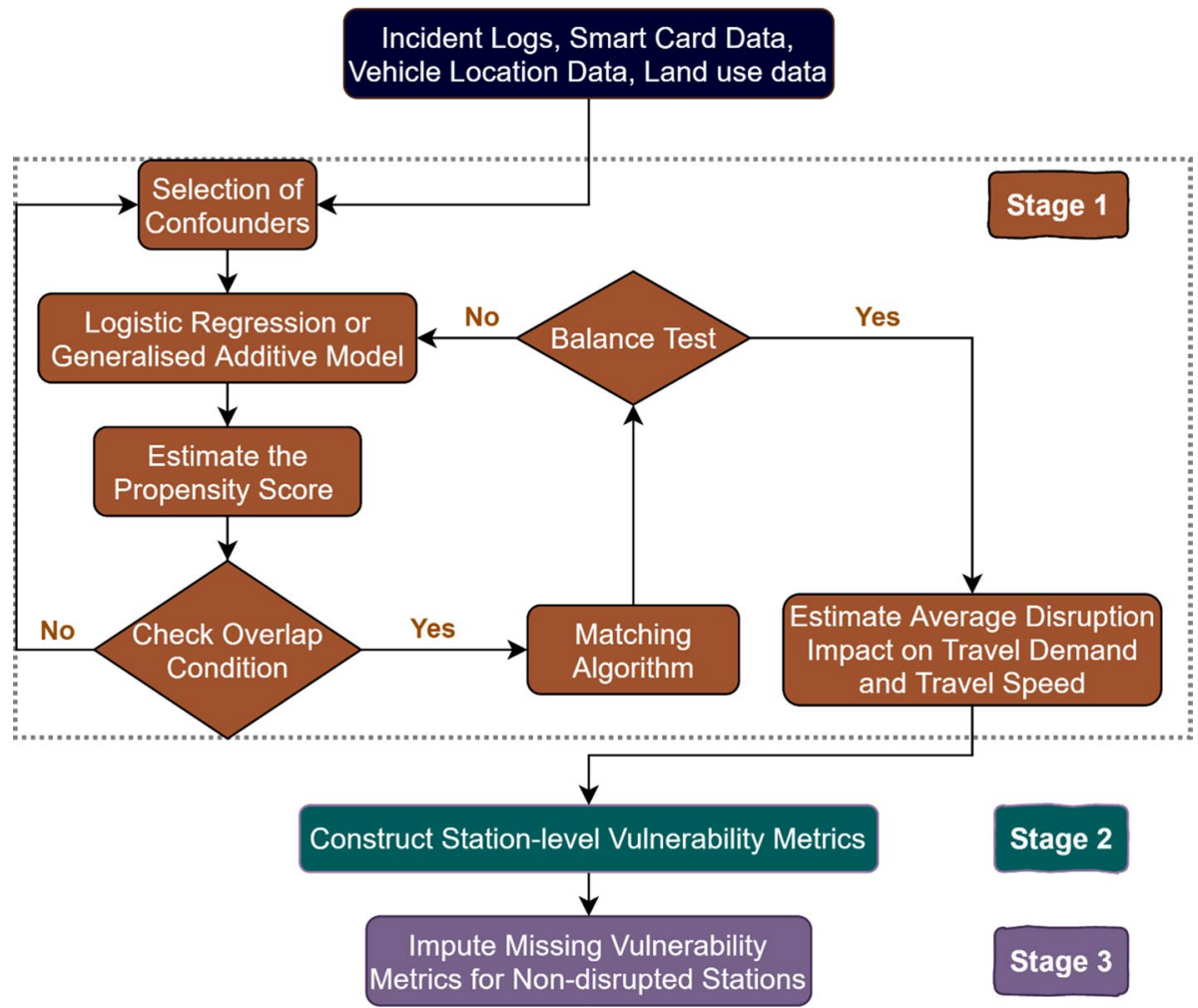

Fig. 1 Flowchart of the paper's methodological framework

\section{Stage 1: Causal inference method to estimate disruption impact}

To evaluate the impact of a disruption on a metro system, we use Rubin's potential outcome framework to establish causality (Rubin 1974). We define metro disruptions as 'treatments' and the objective of our analysis is to quantify the causal effect of treatments on 'outcomes' related to system performance. ${ }^{3}$ Specifically, we are interested in estimating station-level causal effects of disruptions on (1) travel demand, (2) travel speed of passengers, and (3) passenger flow distributions from/to a station. From the literature, we know that factors such as passenger demand, weather conditions, network topology and engineering design influence the likelihood of disruption occurrence (Brazil et al. 2017; Melo et al. 2011; Wan et al. 2015). Therefore, the assignment of the treatment is not random. This is important in our context because the factors associated with the assignment of the treatment are also likely to affect the outcomes of interest, and are thus potential confounders in estimation of impacts. Since previous studies

\footnotetext{
3 In causal inference, 'treatment' means the intervention or exposure assigned to (or encountered by) study units, and 'outcomes' means the observed results or effects of the intervention on a response variable of interest. In the context of this study, service disruptions that occurred at metro stations are the 'treatment', and 'outcomes' are the performance of metro services such as travel demand, journey speed, and passenger flow distribution.
} 
on disruption impact have ignored the non-randomness of treatments, their estimated impact may be biased.

We adopt propensity score matching (PSM) methods to address this issue, which potentially eliminates such confounding biases. The propensity score is defined as the conditional probability that a unit receives treatment given its baseline confounding characteristics. If the observed characteristics sufficiently capture the sources of confounding, then the propensity score can be used to consistently estimate impacts given conditional independence between treatment assignment and outcomes (e.g. conditional on the propensity score) (Imbens and Rubin 2015). This index is obtained by estimating a relationship between treatment assignment and baseline confounding characteristics using a regression model. The estimated propensity score is then used to form various semi-parametric estimators of the treatment effect such as weighting, regression, and matching. In this section, we first provide a contextual formulation of PSM and then describe how we apply PSM to quantify the causal impact of metro disruptions on the performance of metro systems.

\section{Propensity score matching (PSM) methods}

The system-level impact, which averages the impact of all disruptions occurred within the metro system, is too generic to represent network vulnerability. Thus, we focus instead on estimating station-level disruption impacts. We define study unit $i$ as the observation of a metro station within a 15 -min interval. The treatment variable, denoted by $W_{i t} \in\{0,1\}$, records whether study unit $i$ at time $t$ is observed in a disrupted $\left(W_{i t}=1\right)$ or undisrupted state $\left(W_{i t}=0\right)$. To quantify disruption impacts, we define outcomes of interest as the changed travel demand and average speed of trips that start from the given study unit, denoted by $Y_{i t}$.

$$
\begin{aligned}
Y_{i t}\left(W_{i t}\right) & =Y_{i t}(0) \times\left(1-W_{i t}\right)+Y_{i t}(1) \times W_{i t} \\
Y_{i t} & = \begin{cases}Y_{i t}(0) & \text { if } W_{i t}=0 \\
Y_{i t}(1) & \text { if } W_{i t}=1\end{cases} \\
i & =1, \ldots, n \quad t=1, \ldots, T,
\end{aligned}
$$

where $n$ is the total number of stations within the metro system, and $T$ is the total number of time intervals during the study period (for example, $\mathrm{T}=4$ if study period is $1 \mathrm{~h}$ ). $Y_{i t}(0)$ and $Y_{i t}(1)$ are counterfactual potential outcomes, only one of which is observed. The propensity score, denoted by e $\left(X_{i t}\right)$, is obtained by regressing $W_{i t}$ on confounding factors, denoted by $X_{i t}$. We discuss potential confounding factors in the empirical study in "Case study: London underground" section.

To derive valid causal inference using PSM we need our model to satisfy three key assumptions. The first one is the conditional independence assumption (CIA),

$$
W_{i t} \perp\left(Y_{i t}(0), Y_{i t}(1)\right) \mid X_{i t},
$$

which states that conditional on the observed confounding factors $X_{i t}$, the treatment assignment should be independent of the potential outcomes. The advantages of the propensity score stems from a property that this conditional independence can be achieved by just conditioning on a scalar rather than high-dimensional baseline covariates (Rosenbaum and Rubin 1983). Thus, the CIA based on the propensity score can be written as: 


$$
W_{i t} \perp\left(Y_{i t}(0), Y_{i t}(1)\right) \mid \mathrm{e}\left(X_{i t}\right)
$$

The second assumption requires common support in the covariate distributions by treatment status:

$$
0<\operatorname{pr}\left(W_{i t}=1 \mid X_{i t}=x\right)<1 \text { for all } x,
$$

which states that the conditional distribution of $X_{i t}$ given $W_{i t}=1$ should overlap with that of the conditional distribution of $X_{i t}$ given $W_{i t}=0$. This assumption can be tested by comparing the distributions of propensity scores between treatment and control groups.

The third assumption, also known as the stable unit treatment value assumption (SUTVA), requires that the outcome for each unit should be independent of the treatment status of other units (Graham et al. 2014).

If all three assumptions hold and the outcome variable is entry demand or travel speed, the average treatment effect (ATE) of disruptions on a station $i$ can be derived using the following equations (Imbens and Wooldridge 2009):

$$
\begin{gathered}
\tau_{\text {ATE }}^{i}=\hat{\tau}_{\text {match }}^{i}=\frac{1}{T_{d}} \sum_{t=1}^{T_{d}}\left(\hat{Y}_{t}^{i}(1)-\hat{Y}_{t}^{i}(0)\right), \\
\hat{Y}_{t}^{i}(1)=Y_{i t} \\
\hat{Y}_{t}^{i}(0)=\frac{1}{M} \sum_{t_{c} \in J_{M}(i t)} Y_{i t_{c}} \\
i=1, \ldots, n \quad t=1, \ldots, T_{d}
\end{gathered}
$$

where $t \in\left\{1, \ldots, T_{d}\right\}$ denotes all the disrupted time intervals of station $i$ during the study period and $Y_{i t_{c}}$ is the outcome of the control unit $t_{c}$ corresponding to station $i$ disrupted or treated at time $t$. $J_{M}(i t)$ is a set of indices of the closest $M$ control units (in terms of propensity scores) for station $i$ disrupted at time $t$ during the same 15-min interval, but on a different day. ${ }^{4}$ Thus, $\hat{\tau}_{\text {match }}^{i}$ represents the average of the difference between the outcomes of treated and matched control units.

When the outcome variable is trip distribution, ATE can be expressed as:

$$
\begin{gathered}
\tau_{A T E}^{i}=\hat{\tau}_{\text {match }}^{i}=\frac{1}{T_{d}} \sum_{t=1}^{T_{d}}\left[\operatorname{dif}\left(\hat{Y}_{t}^{i}(1), \hat{Y}_{t}^{i}(0)\right)\right], \\
\hat{Y}_{t}^{i}(1)=Y_{i t}=\left(r_{1 i t}^{1}, r_{1 i t}^{2}, \ldots, r_{1 i t}^{k}\right), \\
\hat{Y}_{t}^{i}(0)=\frac{1}{M} \sum_{t_{c} \in J_{M}(i t)} Y_{i t_{c}}=\left[\frac{1}{M} \sum_{t_{c} \in J_{M}(i t)}\left(r_{0 i t_{c}}^{1}\right), \ldots, \frac{1}{M} \sum_{t_{c} \in J_{M}(i t)}\left(r_{0 i t_{c}}^{k}\right)\right], \\
i=1, \ldots, n \quad k=1, \ldots, n \quad t=1, \ldots, T_{d},
\end{gathered}
$$

\footnotetext{
${ }^{4}$ Please note that the study period of this study is 35 days. Therefore, we observe the same station across multiple days (see "Case study: London underground" section for details).
} 
where for a treated or disrupted unit, $Y_{i t}$ denotes the distribution of trips made from (outward) and to (inward) station $i$ at time $t, r_{1 i t}^{k}$ denotes the ridership from the disrupted station $i$ to station $k$ in case of outward flow (or from station $k$ to station $i$ in case of inward flow) at time $t$. Correspondingly, $Y_{i t}$ denotes a composite distribution which averages the ridership distribution of all closest $M$ control units during the same 15-min duration, but on a different day. $r_{0 i t_{c}}^{k}$ denotes the ridership between station $i$ and station $k$ for a non-disrupted period $t_{c}$ in the control group. $\operatorname{dif}(a, b)$ is a function to calculate the distance between discrete distributions $a$ and $b$. In the context of this study, we consider three distance functions:

$$
\begin{gathered}
\operatorname{dif}_{1}\left(\hat{Y}_{t}^{i}(1), \hat{Y}_{t}^{i}(0)\right)=\sqrt{\sum_{k=1}^{n}\left(r_{1 i t}^{k}-\frac{1}{M} \sum_{t_{c} \in J_{M}(i t)}\left(r_{0 i t_{c}}^{k}\right)\right)^{2}}, \\
\operatorname{dif}_{2}\left(P\left(\hat{Y}_{t}^{i}(1)\right), P\left(\hat{Y}_{t}^{i}(0)\right)\right)=\frac{1}{\sqrt{2}} \times \sqrt{\sum_{k=1}^{n}\left(\sqrt{P_{i t}^{k}(1)}-\sqrt{P_{i t}^{k}(0)}\right)^{2}}, \\
\operatorname{dif}_{3}\left(P\left(\hat{Y}_{t}^{i}(1)\right) \| P\left(\hat{Y}_{t}^{i}(0)\right)\right)=\sum_{k=1}^{n}\left[P_{i t}^{k}(1) \times \log \left(\frac{P_{i t}^{k}(1)}{P_{i t}^{k}(0)}\right)\right], \\
P\left(\hat{Y}_{t}^{i}(1)\right)=\left(p_{i t}^{1}(1), \ldots, p_{i t}^{k}(1)\right) \\
P\left(\hat{Y}_{t}^{i}(0)\right)=\left(p_{i t}^{1}(0), \ldots, p_{i t}^{k}(0)\right) \\
p_{i t}^{k}(1)=\frac{r_{1 i t}^{k}}{\sum_{k=1}^{n}\left(r_{1 i t}^{k}\right)}, \\
p_{i t}^{k}(0)=\frac{\frac{1}{M} \sum_{t_{c} \in J_{M}(i t)}\left(r_{0 i t_{c}}^{k}\right)}{\sum_{k=1}^{n}\left(\frac{1}{M} \sum_{t_{c} \in J_{M}(i t)}\left(r_{0 i t_{c}}^{k}\right)\right)}
\end{gathered}
$$

where $\operatorname{dif}_{1}($.$) represents the Euclidean distance, which directly aggregates the difference$ between each element of the input distributions without normalising. The latter two functions compare the probability mass functions $P\left(\hat{Y}_{t}^{i}(1)\right)$ and $P\left(\hat{Y}_{t}^{i}(0)\right)$. dif $f_{2}($.$) represents the$ Hellinger distance and $\operatorname{dif}_{3}($.$) represents Kullback-Leibler divergence (also known as rela-$ tive entropy). Each distance function has its strength and weakness, which we highlight in Sect. 5.4 while discussing results of the empirical study.

In the next subsection, we explain how the causal inference framework introduced in Eqs. (1), (5) and (6) can be implemented in the present application. Following the framework summarised in Fig. 1, we first provide details of the propensity score model, followed by description of our matching algorithms and the estimation of disruption impacts.

\section{Application of PSM methods}

To predict the propensity score, i.e. probability of encountering disruptions at a metro station within 15-min interval conditional on the baseline confounding characteristics, we use the logistic regression model with a linear link function: 


$$
\begin{gathered}
\mathrm{e}\left(X_{i t}\right)=\operatorname{pr}\left(W_{i t}=1 \mid X_{i t}=x^{\{c\}}\right)=p(i t) \\
\log \left[\frac{p(i t)}{1-p(i t)}\right]=\alpha+\beta x^{\{c\}} \quad i=1, \ldots, n \quad t=1, \ldots, T,
\end{gathered}
$$

where $\alpha$ is the intercept and $\beta$ is the vector of regression coefficients related to the vector of confounding factors $x^{\{c\}}$. In our empirical study, a station with a higher number of incidents in the past is more likely to encounter a new disruption in the future, just like the black spot on highways. To account for this temporal correlation among disruption occurrence, we ensure that confounding factors contain the history of past disruptions happened on the same day.

Additionally, we also consider a more advanced generalised additive model (GAM), in which the logarithm of the odds ratio is modelled via semi-parametric smoothing splines. A GAM has potential to uncover flexible relationships between the likelihood of disruption occurrence and confounding factors. The GAM with temporal correlation is presented in Eq. (11):

$$
\begin{gathered}
\mathrm{e}\left(X_{i t}\right)=p r\left(W_{i t}=1 \mid X_{i t}=x^{\{c\}}\right)=p(i t), \\
\log \left[\frac{p(i t)}{1-p(i t)}\right]=\alpha+f\left(x^{\{c\}} ; \beta\right) \quad i=1, \ldots, n \quad t=1, \ldots, T,
\end{gathered}
$$

where $f\left(x^{\{c\}} ; \beta\right)$ is a flexible spline function of baseline characteristics. After estimating propensity scores, we check the common support (overlap) assumption to ensure the effective matching and reliability of the propensity score estimates (Lechner 2001).

The next step is matching. Every treated unit $i$ at time $t$ is paired with $M$ similar control units based on the value of their propensity scores and time-of-day characteristics. Since there is no theoretical consensus on the superiority of matching algorithms, we adopt two commonly used approaches: Subclassification Matching and Nearest Neighbour Matching. We then compare them with different replacement conditions and pairing ratios and select the one that balances the greatest disparity among the mean of confounding factors. It is also necessary to check the conditional independence assumption after matching. We conduct balancing tests to check whether the disrupted units and the matched units are statistically similar across the domain of confounders. If significant differences are found, we try another specification of the propensity score model and repeat the above-discussed procedure.

In the last step, we estimate station-level disruption impact using Eqs. (5) and (6). Given the matched pairs, the treatment effect for a station at a specific period is estimated as the difference between outcomes of the treated unit and its matched control units. Then the average station-level disruption impact is obtained by averaging these differences across all disrupted periods. We separately estimate the average treatment effects for three measures of metro performance:

1. Entry ridership: the number of passengers who enter the study unit.

2. Average travel speed: average of the speed of all trips that start from the study unit. For each trip, speed is computed as travel distance divided by observed journey time. Whereas journey time is directly obtained using the smart card data, travel distance 
(track length) of the most probable route is derived using the shortest path algorithm. ${ }^{5}$ Passengers who had left the system and used other transport modes to reach the final destination are not included in the computation of this metrics. If the origin station is entirely closed and no passenger can continue trips by metro, then the average speed will be zero. If the origin station is partially closed, this metrics reflects the average speed of passengers who remain in the system.

3. Distribution of passenger flow: the distribution of completed trips that start from (outward flow) and arrive to (inward flow) the study units.

\section{Stage 2: Constructing vulnerability metrics}

We propose four station-level vulnerability metrics that are constructed from the empirical estimates of disruption impacts on the above-discussed performance measures.

(i) The loss of travel demand is expressed as:

$$
d_{i}=-\tau_{A T E}^{i}(\text { entry }),
$$

where $\tau_{A T E}^{i}$ (entry) (calculated using Eq. 5) denotes the station-level change in the number of entry passengers due to service disruptions. $d_{i}$ is the loss of demand from external passengers who have not entered the metro system during a 15-min interval due to disruption.

(ii) The loss of average travel speed quantifies the decline in level of service experienced by each passenger at a metro station (individual delay), which is expressed as:

$$
s_{\text {avg }}^{i}=\tau_{\text {ATE }}^{i}(\text { speed }),
$$

where $\tau_{A T E}^{i}$ (speed) (calculated using Eq. 5) denotes the decrease in average travel speed of trips starting from station $i$ during a 15-min disruption period. By definition, $s_{\text {avg }}^{i}$ accounts for the changes in both travel distance and journey time of passengers.

(iii) The loss of gross travel speed reflects the loss of passenger kilometres per unit time, which is expressed as:

$$
s_{\text {gross }}^{i}=\tau_{A T E}^{i}(\text { speed }) \times r_{i},
$$

where $r_{i}$ denotes the average entry ridership of all disrupted 15-min intervals at the corresponding station. Thus, $s_{\text {gross }}^{i}$ denotes the total decrease in average travel speed for all passengers who start their journeys from station $i$ during a 15-min service disruption.

(iv) The irregularity in passenger flow reflects the degree of deviation in the distribution of trips from/to the disrupted station as compared to regular conditions, which is expressed as:

$$
f_{i}=\tau_{A T E}^{i}(\text { flow })
$$

\footnotetext{
${ }^{5}$ For future research, conditional on the availability of vehicle location data, the shortest path algorithm can be replaced by the passenger-train assignment algorithm (Hörcher et al. 2017; Zhu and Goverde 2019) to infer the most likely path chosen by passengers.
} 
where $\tau_{A T E}^{i}($ flow) (calculated using Eq. 6) denotes the average irregularity in flows that start from or arrive at station $i$ during a 15-min disruption period. This metrics extends the scope of vulnerability measurement in terms of the entire distribution of entry/exit ridership, instead of just analysing the disruption impact on the entry or exit demand (that is, moments of the trip distribution).

\section{Stage 3: Imputing missing vulnerability metrics}

Some stations may not encounter any incidents within the study period. Thus, the empirical disruption impact and the vulnerability metrics cannot be estimated directly for these stations. To predict the missing metrics of non-disrupted stations, we estimate a random forest regression model (Hastie et al. 2009):

$$
\hat{f}_{r f}^{B}\left(x^{\{s\}}\right)=\frac{1}{B} \sum_{b=1}^{B} T\left(x^{\{s\}} ; \theta_{b}\right),
$$

where $\hat{f}_{r f}^{B}\left(x^{\{s\}}\right)$ denotes the random forest predictor. In the equation above, $B$ is the number of trees, $x^{\{s\}}$ is a vector of input features (see Table 2 for details). Furthermore, $T\left(x^{\{s\}} ; \theta_{b}\right)$ is the output of the $b$ th random forest tree, and $\theta_{b}$ characterizes the $b$ th random forest tree. The random forest regression that we apply here is a combination of a bagging algorithm and ensemble learning techniques. By averaging the output of several trees (or weak learners in boosting terminology), it reduces the overfitting problem.

For this study, random forest (RF) is an appropriate prediction method. Interested readers are referred to Hastie et al. (2009) for details of RF regression algorithms, who explain the reasons behind its superior prediction accuracy as compared to other competing machine learning methods (Khalilia et al. 2011; Couronné et al. 2018). However, considering that the field of machine learning is evolving rapidly, we also encourage readers to explore state-of-the-art alternatives to RF and test different prediction algorithms to find the most suitable algorithm for their data.

\section{Case study: London underground}

In 2013, the London Underground (LU) had 270 stations and 11 lines, with a total length of $402 \mathrm{~km}$ stretching deep into Greater London. The circle-radial network structure, as shown in Fig. 2 (Wikimedia Commons 2013), is one of the largest and most complex metro systems in the world. Of all lines within the network, one is circular (Circle Line) covering Central London, and the remaining 10 are radial routes converging at the centre of the system. For connectivity among stations, LU has 56 stations connecting 2 lines, 16 stations connecting 3 lines and 8 stations connecting more than 4 lines. LU is also one of the busiest metro systems, with 1.265 billion journeys by the end of 2013 (Transport for London 2019). Due to over 150 years old operations and enormous passenger demand, disruptions occur frequently in LU.

We use the following data to analyse the station-level vulnerability of the LU system. We conducted data processing and analysis using open-source R software (version 4.0.3).

Pseudonymised smart card data Transport for London (TfL) provided automated fare collection data from 28/10/2013 to 13/12/2013 (35 weekdays) between 6:00 and 24:00. 


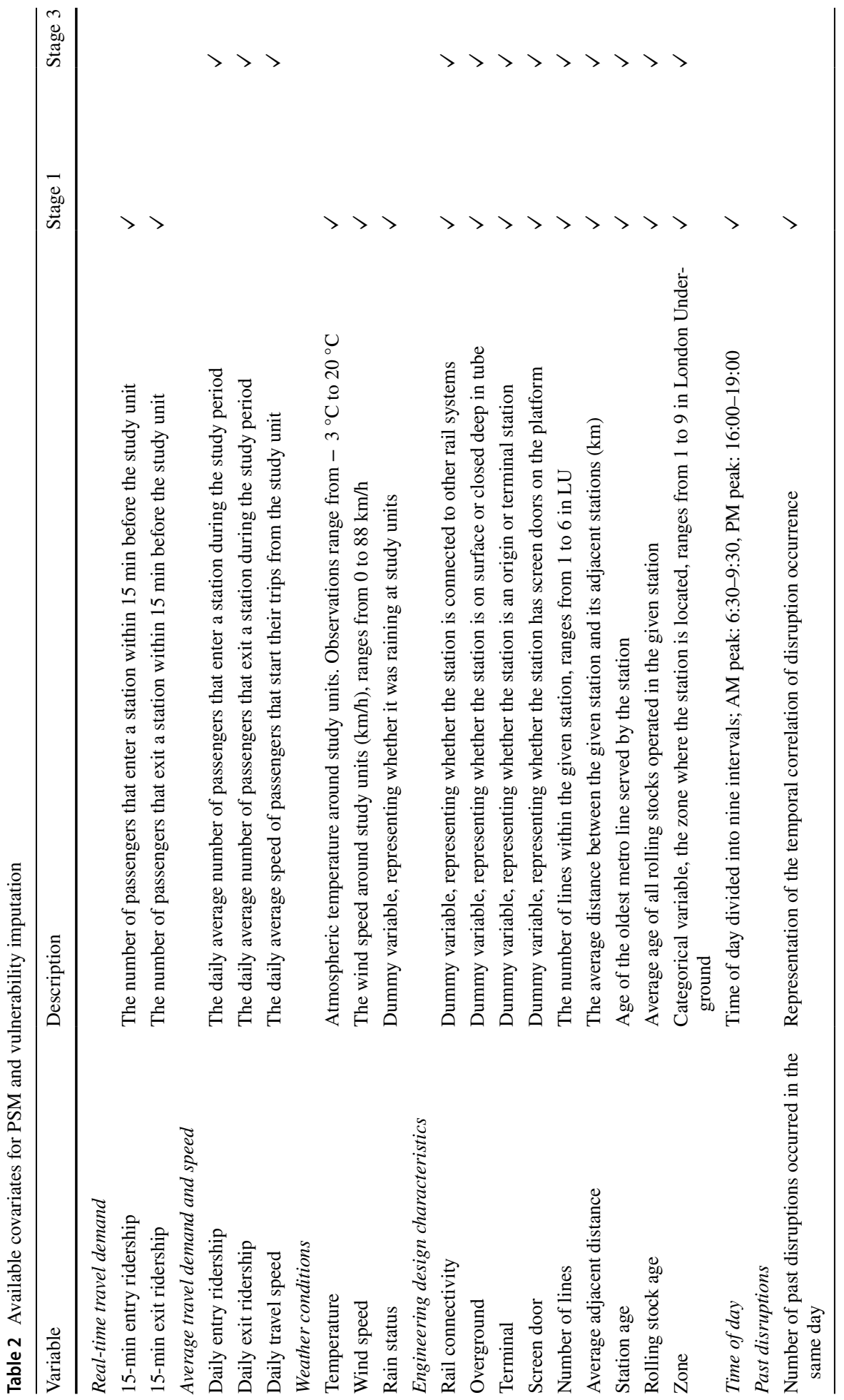




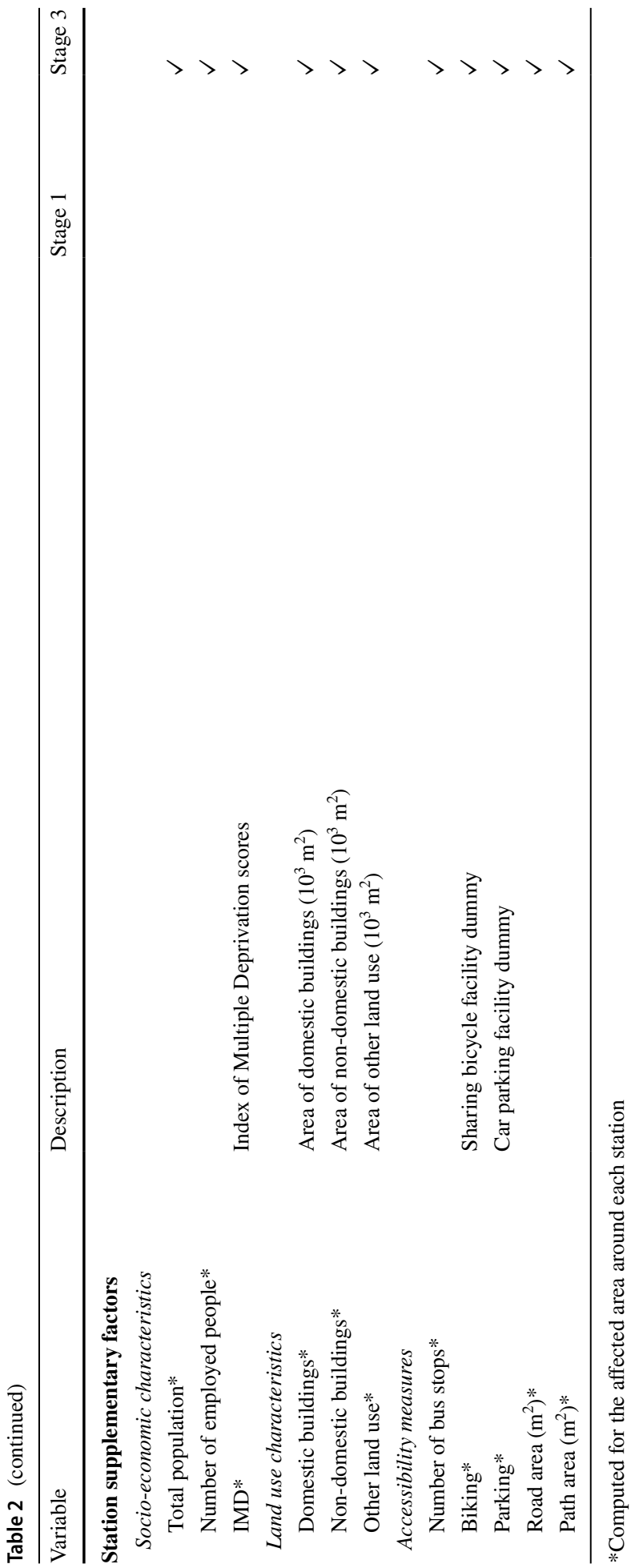




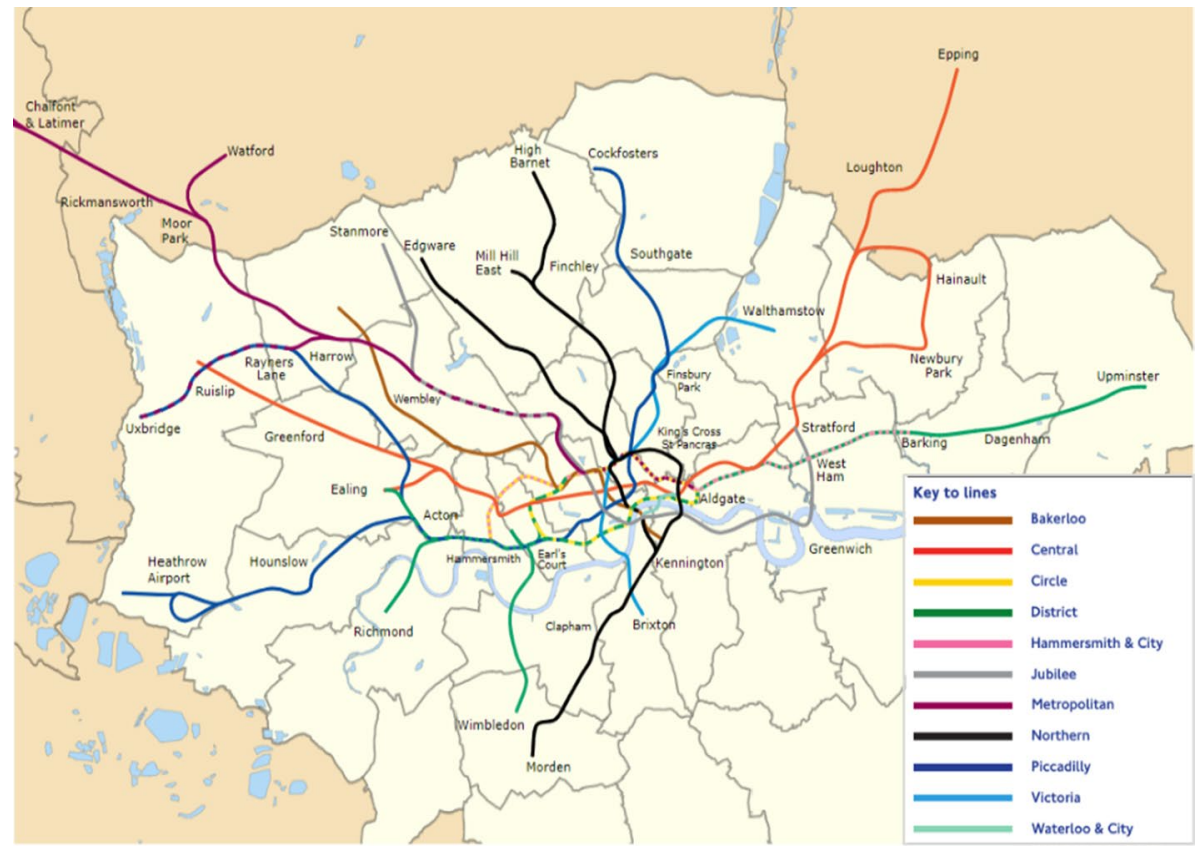

Fig. 2 London Underground network. Adapted from (Wikimedia Commons, 2013)

We consider this duration as our study period. The smart card data contain information on transaction date and time, entry and exit locations, encrypted card ID and ticket type (pay as you go/season ticket). The resolution of time stamps exacts to $1 \mathrm{~min}$. By using smart card data, we compute entry/exit ridership of each station and obtain passengers' journey time and travel speed.

Incidents and service disruption information $\mathrm{TfL}$ also provided incident information data for our study period. By mining provided incidents logs, we construct an accurate database of service disruptions, which includes the occurrence time, location and duration of disruptions.

LU network topology information We collect data on station coordinates, topology structure and the length of tracks between adjacent stations from open databases authorised by TfL. ${ }^{6}$

Weather data We collect temperature $\left({ }^{\circ} \mathrm{C}\right)$, wind speed $(\mathrm{km} / \mathrm{h})$ and rain status from the Weather Underground web portal. ${ }^{7}$ Based on the observations of over 1000 weather stations around London, we estimate weather conditions for all LU stations at 15-min resolution for our study period.

\footnotetext{
6 Source: https://www.whatdotheyknow.com.

7 Weather information web portal: https://www.wunderground.com/.
} 


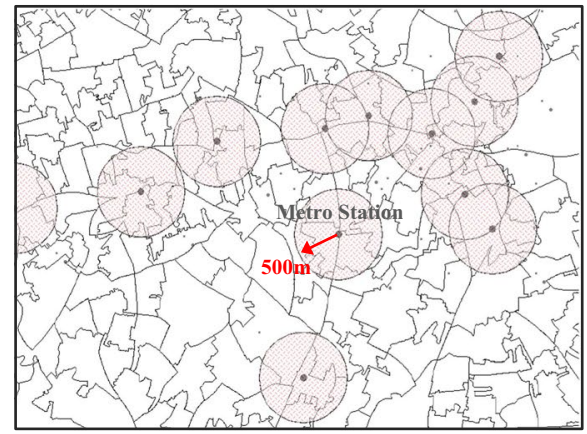

(a) Station affected areas

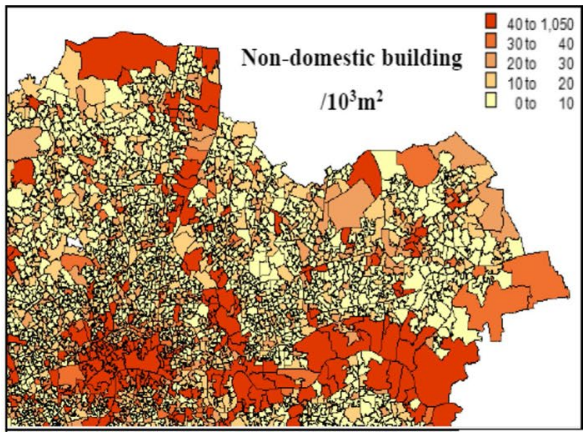

(b) An example of LSOA data

Fig. 3 The illustration of calculating station-level supplementary factors

LU station characteristics These station-level features include daily ridership, station age, rolling stock age, sub-surface/deep-tube stations, terminal stations and screen doors. We also calculate supplementary factors, which capture the characteristics of the affected areas around metro stations. To compute these factors, we define the affected area as a circular area with the radius of 500 metres around the station. We use 2011 UK Census data at Lower Super Output Area (LSOA) level $^{8}$ to calculate these supplementary factors. We select all LSOAs whose centroids are within the 500 metres radius of the affected area. We then average the related statistics of the selected LSOAs according to their areas in the circle. Figure 3 illustrates the above process of calculations.

To construct the causal inference framework for LU, our study unit is the observation of metro stations during each 15-min interval within the system service time. We define metro disruption as the state when scheduled train services are interrupted for at least $10 \mathrm{~min}$ at a station. Over the study period, LU encountered 2894 disruptions lasting from $10 \mathrm{~min}$ to $11 \mathrm{~h}$. The aim of causal inference is to estimate the unbiased impact of these observed disruptions (i.e., treatment) on system-performance measures (outcome). The treatment status $W_{i t}$ is constructed according to the disruption database mentioned in "Case study: London underground" section. To match the disruption duration with the timeframe of study units, we define the following rule to assign the treatment status: if a disruption occurs within a 15-min interval $t$ of a given station $i$, we regard this study unit as disrupted (i.e., $W_{i t}=1$ ), no matter whether disruptions start or end in the middle or last for the entire 15-min interval. Conversely, if the station is under normal service during entire 15-min interval, we regard this study unit as un-disrupted (i.e., $W_{i t}=0$ ). The treatment outcomes $Y_{i t}$ are presented as three station-level performance indicators: entry ridership, exit ridership, and average travel speed.

As discussed earlier, metro disruptions may not occur randomly. We list all potential confounding factors for LU in Table 2, which we use in estimating the propensity score model (Sect. 3.1). These confounders are selected according to the literature and expertise, including travel demand, weather conditions, engineering design, time of day and past disruptions (Brazil et al. 2017; Melo et al. 2011; Wan et al. 2015). Table 2 also

\footnotetext{
8 Source: London Datastore, published by Greater London Authority: https://data.london.gov.uk/census/.
} 
shows available covariates for the imputation of missing vulnerability metrics in Stage 3 (Sect. 3.3), which not only include some of confounders, but also include supplementary factors of LU station characteristics.

\section{Results and discussions}

Out of 270 stations of the LU system, TfL provided the required datasets for 265 stations during the study period (28/10/2013-13/12/2013). Smart card data were missing for the remaining five stations. Our analysis only covers weekdays, during which the system is open for $18 \mathrm{~h}$ per day, starting from 6:00 a.m. to midnight. Based on the assumption of exchangeability of weekdays (Silva et al. 2015), we generate a panel dataset with a total of $265 \times 35 \times 18 \times 60 / 15=667,800$ study units. Although the PSM method is a data-hungry method, the untreated pool (control group) is large enough to ensure adequate matches for treated units. Specifically, the ratio of the number of control and treatment units is around $15: 1$.

\section{Propensity score models}

We initially include three key baseline covariates - past disruptions, time of day and realtime travel demand-in the logistic regression. We then iteratively add one of the remaining covariates at a time from covariates listed in Table 2 and conduct the likelihood ratio test to decide whether the additional covariate should be included in the final specification or not. We also test Generalised Additive Models (GAM), but we do not observe any gains in the model fit. A high proportion of dummy variables (11 out of 19) may limit the gains from a flexible spline specification of the link function. The estimation results of the logistic regression model are summarised in Table 3.

The role of propensity score models is to establish a comprehensive index to represent all confounding factors, rather than predicting treatment assignment. While noting that the logistic regression model does not reveal the causal effect of covariates on the likelihood of incident occurrence, we succinctly discuss the multivariate correlations uncovered by this model. The coefficients of time dummies indicate that incidents are more likely to occur in morning peak hours. Positive signs on coefficients of the remaining confounders (except Rail dummy) confirm that all these factors increase the probability of encountering a disruption. Specifically, surface stations are more susceptible to the surrounding environment than those in tubes. We find statistically significant interaction effects between wind speed and Overground dummy. The accumulated number of past disruptions happened on the same day increases the probability of encountering another incident. Conclusively, the propensity score model reveals that the occurrence of metro disruptions is non-random, which, in turn, also justifies the application of causal inference methods in estimating disruption impacts.

Alternatively, the estimated propensity score model can also be viewed as a binary classifier that predicts whether metro disruptions occur or not. To illustrate its diagnostic ability, we compute the area under the receiver operating characteristic curve: AUC $=0.796$, which again indicates that the occurrence of metro disruptions is non-random. 
Table 3 The results of propensity score model (logistic regression)

\begin{tabular}{|c|c|c|}
\hline Confounders & Coef. & SE \\
\hline Intercept & $-4.547 * * *$ & 0.036 \\
\hline Past disruptions & $0.271 * * *$ & $1.634 \mathrm{e}-03$ \\
\hline Time0 (6:00-6:30) (1) & $1.883 * * *$ & 0.027 \\
\hline Time1 (6:30-7:45) (1) & $1.631 * * *$ & 0.021 \\
\hline Time2 (7:45-8:45) (1) & $1.607 * * *$ & 0.022 \\
\hline Time3 (8:45-9:30) (1) & $1.252 * * *$ & 0.026 \\
\hline Time4 (9:30-16:00) (1) & $0.801 * * *$ & 0.016 \\
\hline Time5 (16:00-17:15) (1) & $0.224 * * *$ & 0.026 \\
\hline Time6 (17:15-18:15) (1) & $0.193 * * *$ & 0.028 \\
\hline Time7 (18:15-19:00) (1) & $0.438 * * *$ & 0.029 \\
\hline Temperature $\left({ }^{\circ} \mathrm{C}\right)$ & $0.035^{* * *}$ & $1.926 \mathrm{e}-03$ \\
\hline Wind speed $(\mathrm{km} / \mathrm{h})$ & $0.017 * * *$ & $1.853 \mathrm{e}-03$ \\
\hline Rain (1) & $0.329 * * *$ & 0.015 \\
\hline Rail (1) & $-0.179 * * *$ & 0.013 \\
\hline Overground (1) & $0.219 * * *$ & 0.023 \\
\hline Ave distance $(\mathrm{km})$ & $0.042 * * *$ & $4.748 \mathrm{e}-03$ \\
\hline Station age $(\max )$ & $5.714 \mathrm{e}-04 * *$ & $2.005 \mathrm{e}-04$ \\
\hline Pre 15 -min entry ridership & $1.969 \mathrm{e}-04 * * *$ & $2.098 \mathrm{e}-05$ \\
\hline Rolling stock age (mean) & $4.514 \mathrm{e}-03^{* * *}$ & $4.666 \mathrm{e}-04$ \\
\hline Overground $*$ Wind speed & $0.014 * * *$ & $2.352 \mathrm{e}-03$ \\
\hline McFadden's pseudo R-squared & 0.184 & \\
\hline
\end{tabular}

(1) represents dummy variables

The base dummy for time of the day is Time8 (19:00-24:00)

${ }^{*} p<0.1 ; * * p<0.05 ; * * * p<0.01$

\section{Matching results}

Before the estimated propensity scores are utilised for matching, we inspect the common support condition (assumption 2 of the PSM method). Figure 4 presents the propensity score distributions for both disrupted and normal observations. The histograms display apparent overlap between the treatment and control groups, even for large propensity scores. There is no treated unit outside the range of common support, which means we do not need to discard any observations. We thus conclude that the overlap assumption is tenable in our empirical study.

The PSM method aims to balance the distribution of confounders between the treatment and control groups after the matching stage. To assess the quality of matching, we perform balance tests for four algorithms: subclassification matching, nearest neighbour matching without replacement $(M=1)$, nearest neighbour matching with replacement $(M=1)$ and nearest neighbour matching with replacement $(M=2)$, where $\mathrm{M}$ is the number of matched control units for each treatment unit. It is worth noting that the proposed matching scheme not only conditions on the estimated propensity scores, but also condition on the time-ofday of the treatment (disruption). We find that nearest neighbour matching with replacement $(M=2)$ performs the best, improving the overall balance of all confounding factors by $99.95 \%$. This improvement indicates that within matched pairs, the difference of 


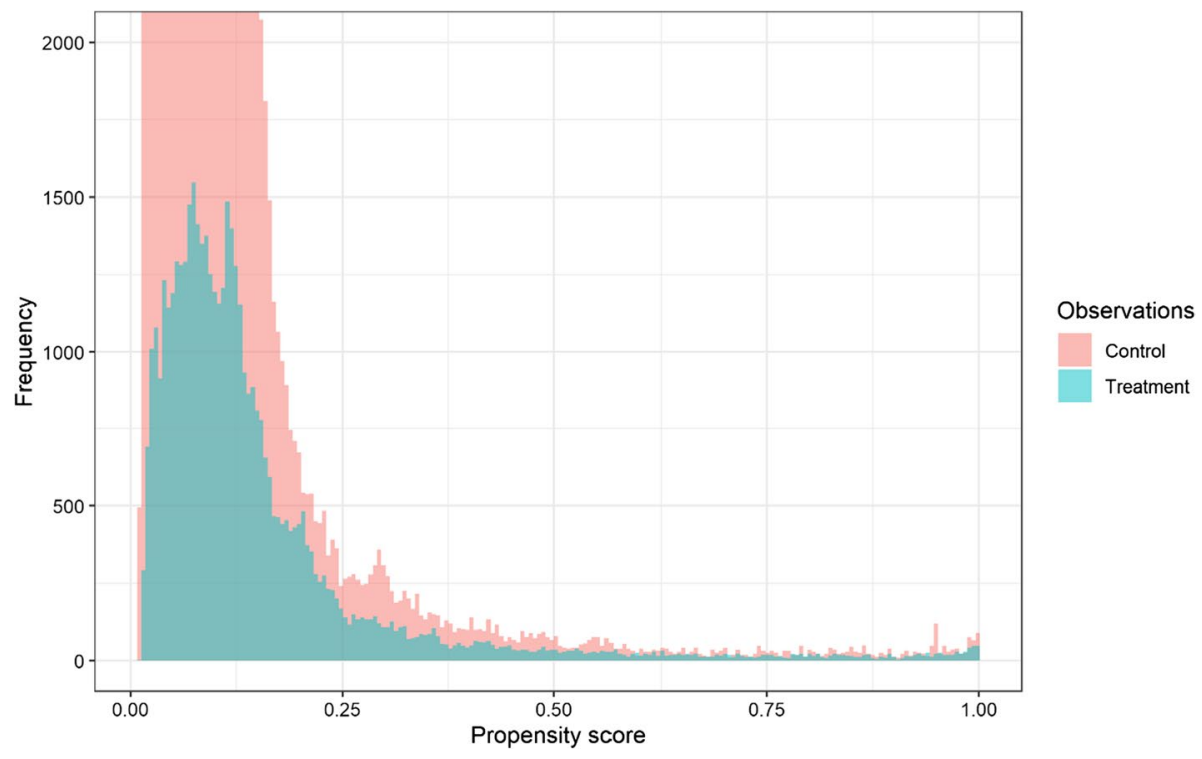

Fig. 4 Histogram of propensity scores to test the common support condition (Due to higher share of the control group, the frequency in Fig. 4 ranges up to 60,000 for lower propensity scores. However, we truncate frequency at 2000 to clearly show the validity of overlap condition across the entire domain of the propensity score.)

propensity scores and time-of-day characteristics between treatment and control units has been reduced by $99.95 \%$, compared with the original data before matching.

\section{Imputation of missing vulnerability metrics}

During the study period, 21 out of 265 stations did not encounter any service disruptions. We apply the random forest regression model to predict the missing vulnerability metrics of these stations. The input features of the model are indicated in "Stage 3" column of Table 2, consisting of station-level supplementary factors and a subset of confounding factors. For each vulnerability metrics, we estimate the random forest regression model using the 'randomForest' package of R (Liaw and Wiener 2002). In terms of model settings, we consider the maximum number of trees to be 5000, randomly sample seven variables as candidates at each split, and assume the minimum size of terminal nodes to be two. The results show that more than $67 \%$ of the variance can be explained by input features for all vulnerability metrics. We summarize the prediction performance of random forest regression in Table 4 and benchmark it against two competing methods: linear regression and support vector machines.

Four measures are considered to benchmark the performance of random forest regression against other methods-mean absolute error (MAE), root mean squared error (RMSE), relative absolute error (RAE), and relative squared error (RSE). Whereas MAE measures the average magnitude of the errors in predictions, RMSE represents the standard deviation of the unexplained variance (Willmott and Matsuura 2005). A better prediction model produces lower values of these performance measures. The results in Table 4 
Table 4 Prediction accuracy of different regression methods

\begin{tabular}{|c|c|c|c|c|}
\hline \multirow[t]{2}{*}{ Vulnerability metrics } & \multirow{2}{*}{$\begin{array}{l}\text { Performance } \\
\text { measures }\end{array}$} & \multicolumn{3}{|c|}{ Imputation methods } \\
\hline & & Random forest & Linear regression & $\begin{array}{l}\text { Support vec- } \\
\text { tor machines }\end{array}$ \\
\hline \multirow[t]{4}{*}{ Demand loss } & MAE & 2.794 & 33.089 & 5.181 \\
\hline & RMSE & 4.285 & 37.342 & 9.766 \\
\hline & RAE & 0.29 & 44.556 & 0.538 \\
\hline & RSE & 0.095 & 330.181 & 0.493 \\
\hline \multirow[t]{4}{*}{ Avg. travel speed loss } & MAE & 0.236 & 11.081 & 0.468 \\
\hline & RMSE & 0.684 & 16.848 & 1.892 \\
\hline & RAE & 0.318 & 1.151 & 0.63 \\
\hline & RSE & 0.111 & 1.468 & 0.848 \\
\hline \multirow[t]{4}{*}{ Gross travel speed loss } & MAE & 62.416 & 979.91 & 114.554 \\
\hline & RMSE & 96.461 & 1224.723 & 216.472 \\
\hline & RAE & 0.314 & 4.932 & 0.577 \\
\hline & RSE & 0.107 & 17.18 & 0.537 \\
\hline \multirow[t]{4}{*}{ Irregularity in flow (Euclidean-entry) } & MAE & 1.405 & 3.514 & 2.213 \\
\hline & RMSE & 1.935 & 4.575 & 3.474 \\
\hline & RAE & 0.23 & 0.574 & 0.362 \\
\hline & RSE & 0.058 & 0.326 & 0.188 \\
\hline \multirow[t]{4}{*}{ Irregularity in flow (Hellinger-entry) } & MAE & 0.02 & 0.051 & 0.034 \\
\hline & RMSE & 0.025 & 0.064 & 0.048 \\
\hline & RAE & 0.246 & 0.625 & 0.417 \\
\hline & RSE & 0.066 & 0.418 & 0.234 \\
\hline \multirow[t]{4}{*}{ Irregularity in flow (KL-entry) } & MAE & 0.276 & 0.498 & 0.333 \\
\hline & RMSE & 0.184 & 0.72 & 0.613 \\
\hline & RAE & 0.241 & 0.654 & 0.436 \\
\hline & RSE & 0.074 & 0.506 & 0.366 \\
\hline
\end{tabular}

indicate that the random forest regression outperforms other competing methods with the lowest MAE, RMSE, RAE and RSE for all vulnerability metrics.

\section{LU vulnerability metrics}

The estimated vulnerability metrics vary across stations in the LU system. We first discuss results for loss of entry demand, loss of average travel speed, and loss of gross travel speed metrics. For 265 operated stations in 2013, during a 15-min period of service disruption, the loss of station entry demand ranges from 0 to 103.4 passengers, the loss of average travel speed ranges from 0 to $21.76 \mathrm{~km} / \mathrm{h}$, and the loss of gross travel speed ranges from 0 to 2032.3 passenger- $\mathrm{km} / \mathrm{h}$. The spatial distributions of these vulnerability metrics are visualised in Fig. 5a-c. For the demand loss and gross speed loss, the large proportion of vulnerable stations are in inner London areas, while a small number of vulnerable stations are also located in suburban areas. Conversely, for the loss of average travel speed, the most vulnerable stations are scattered around outer London areas. These stations usually have only one metro line (internal alternatives) and have 
Fig. 5 Spatial distribution of station-level vulnerability metrics in London underground

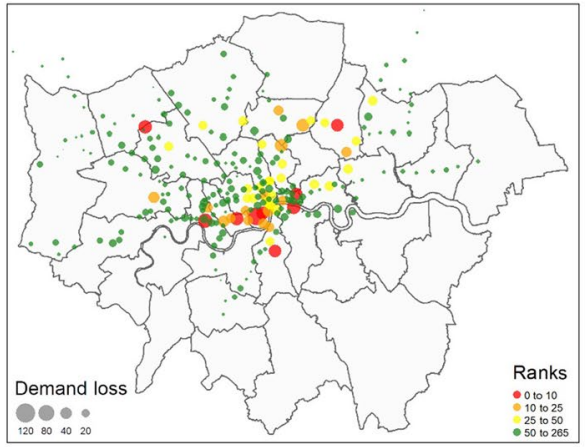

(a) The loss of travel demand

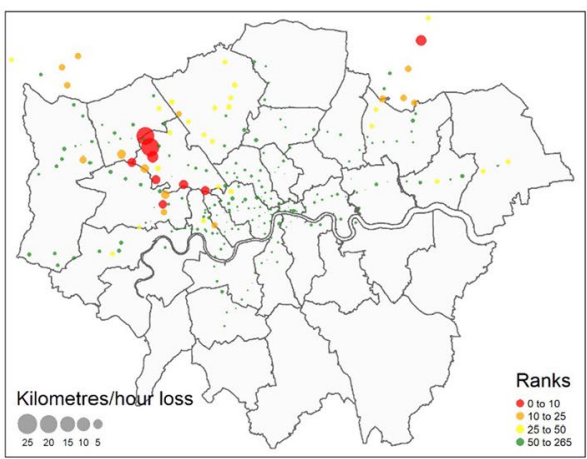

(b) The loss of average travel speed

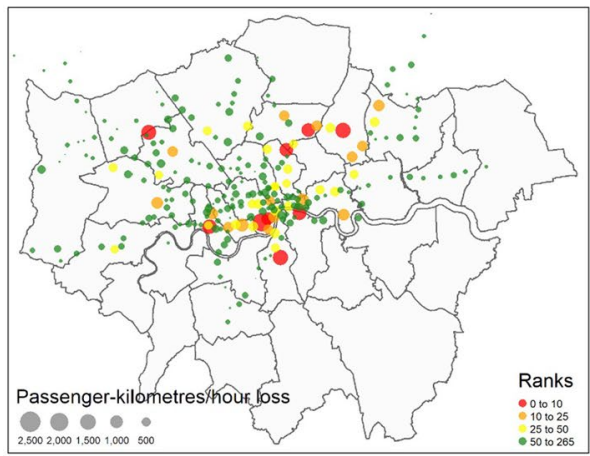

(c) The loss of gross travel speed

very limited access to other transport modes (external alternatives) compared to Central London areas. When passengers encounter disruptions, to continue their trips they need to wait for longer time in the system until train services are recovered. In other words, due to of lack of alternative routes, ${ }^{9}$ passengers at these stations tend to experience more individual delays.

\footnotetext{
9 There can be two types of alternative routes under disruptions-within the metro system (interchange to use other operated lines) and outside of it (in the form of other modes).
} 

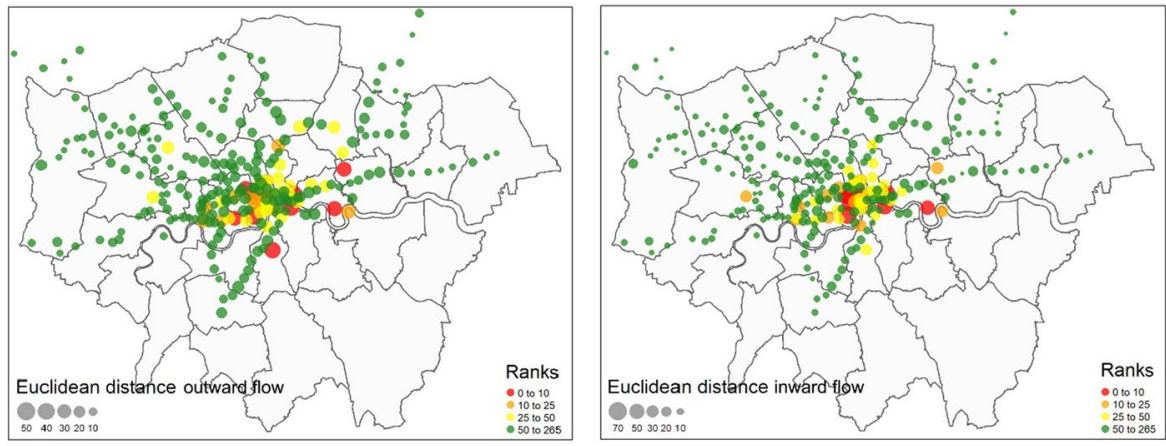

(d) The irregularity in flow distribution from/to the disrupted station (Euclidean distance)
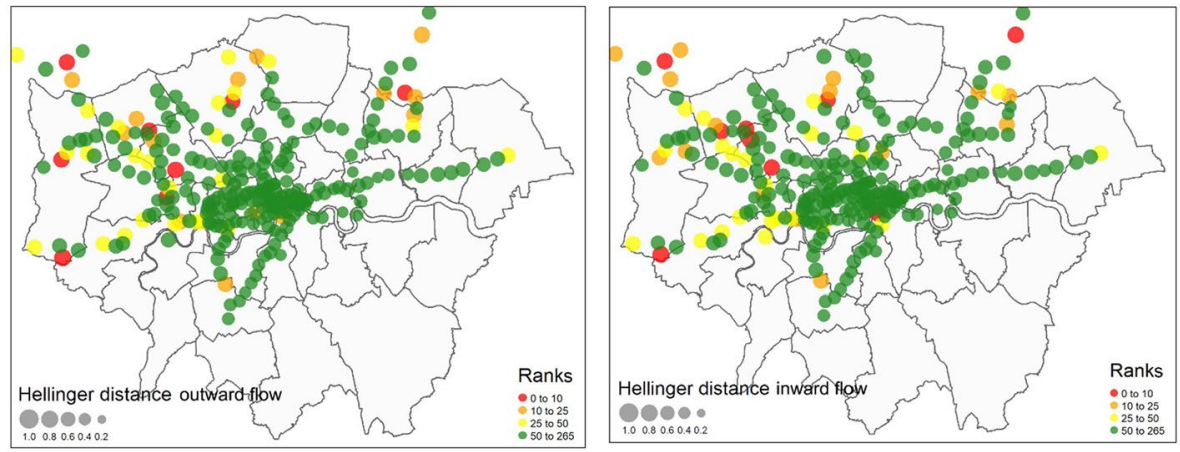

(e) The irregularity in flow distribution from/to the disrupted station (Hellinger distance)
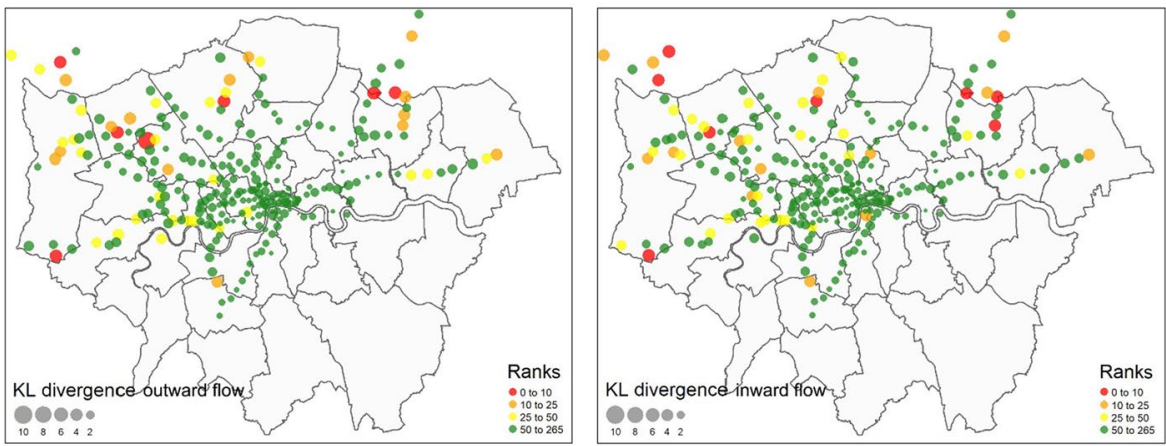

(f) The irregularity in flow distribution from/to the disrupted station (KL divergence)

Fig. 5 (continued)

We firstly sort all 265 stations based on demand and speed loss metrics, and the top 15 stations are presented in Table 5. Victoria is the most vulnerable station based on demand loss and gross speed loss metrics. Other stations such as Hammersmith, London Bridge, Kenton, Brixton are also among the top vulnerable stations based on both metrics. However, based on only the loss of average travel speed metrics, the most vulnerable stations are South Kenton, Kenton and North Wembley in outer London areas, where each passenger suffers the longest delay due to lack of alternative routes. The above rankings based 


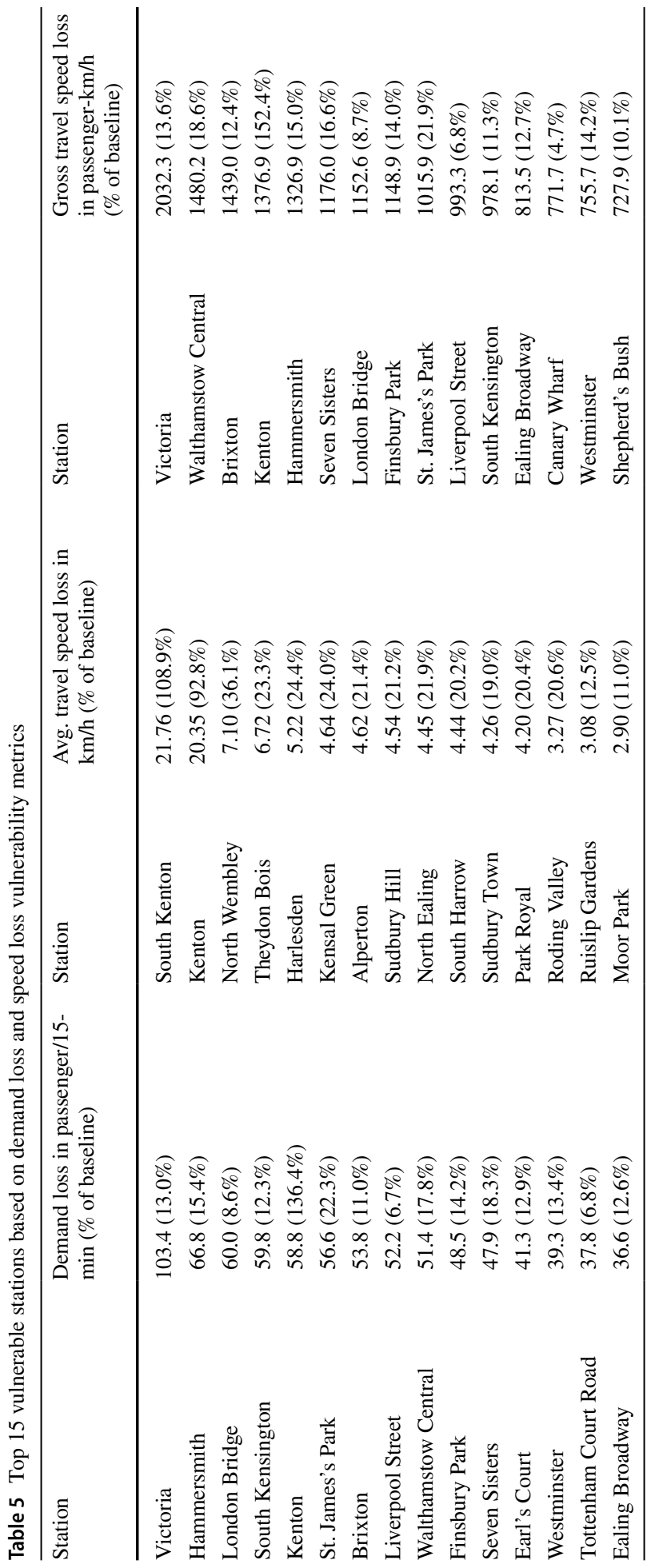


on different vulnerability metrics can assist metro operators in preparing effective plans for ridership evacuation and service recovery.

Table 5 also presents normalised vulnerability metrics for these top 15 stations, which is the relative percentage change as compared to the undisrupted performance measure (baseline). Note that all baseline situations for these three metrics are calculated by using average across undisrupted observations. We find that the rankings based on relative vulnerability metrics can be different than those based on absolute metrics, especially for the loss of travel demand. In more isolated parts of the network, where alternative routes may not be available, stations can lose up to $136.4 \%$ of their normal demand due to service interruption (e.g. Kenton Station in Zone 4 with no intersection metro line). This implies that more connected stations are actually less vulnerable in this respect, as passenger can find alternative routes if one of the lines becomes disrupted. This result also highlights potentially important distinctions about the interpretation of the proposed metrics. In terms of relative metrics of average travel speed, the same top three vulnerable stationsSouth Kenton, Kenton and North Wembley-experienced decrease in average travel speed by $108.9 \%, 92.8 \%$ and $36.1 \%$, respectively, due to disruption. Kenton station is also the most vulnerable stations based on the relative loss of gross trip speed, which is reduced by $152.4 \%$.

We propose three distance measures for the irregularity in flow metrics: Euclidean distance (ED), Hellinger distance (HD) and Kullback-Leibler (KL) divergence for both outward (from) and inward (to) flows. Euclidean distance directly compares the difference of each element of the trip distribution, where the element represents the ridership between a specific station and the disrupted station. ED reflects changes in the magnitude as well as the proportion of the flow of each element because it is not normalised. HD and KL divergence are normalised measures as they compare the difference between probability mass function of trip distributions, which capture only change in the proportion of trips completed between the disrupted and other stations. Unlike ED, HD and KL divergence would not be useful measures if disruption leads to a decrease in ridership across all stations by the same proportion. HD and KL divergence are close in principle, but the latter can be interpreted as the change in relative entropy, which is meaningful in the context of disruptions in metro systems. As an analogy with the concept of entropy in thermodynamics, we may interpret the extra entropy in metro systems as an additional generalised cost (in terms of time and congestion costs) that passengers have to pay under disruptions.

We plot the spatial distribution of all these distance measures in Fig. 5d-f. We also sort all 265 stations based on ED, HD and KL divergence, and the top 15 vulnerable stations are presented in Table 6. We find that the station rankings for outward flow (i.e., the entry ridership distribution) based on ED are similar to those obtained based on demand loss and gross speed loss metrics. They also share a similar spatial distribution of vulnerable stations. As for the distribution of inward flow (i.e., the exit ridership distribution), the most affected stations are mostly busy stations in Central London areas. As expected, station rankings based on HD and KL divergence are similar. For both inward (exit) and outward (entry) flow distributions, suburban stations are more severely affected than Central London stations on a normalised scale. The top 3 stations based on HD and KL divergence are South Kenton, Chesham and Heathrow Terminal 4. 


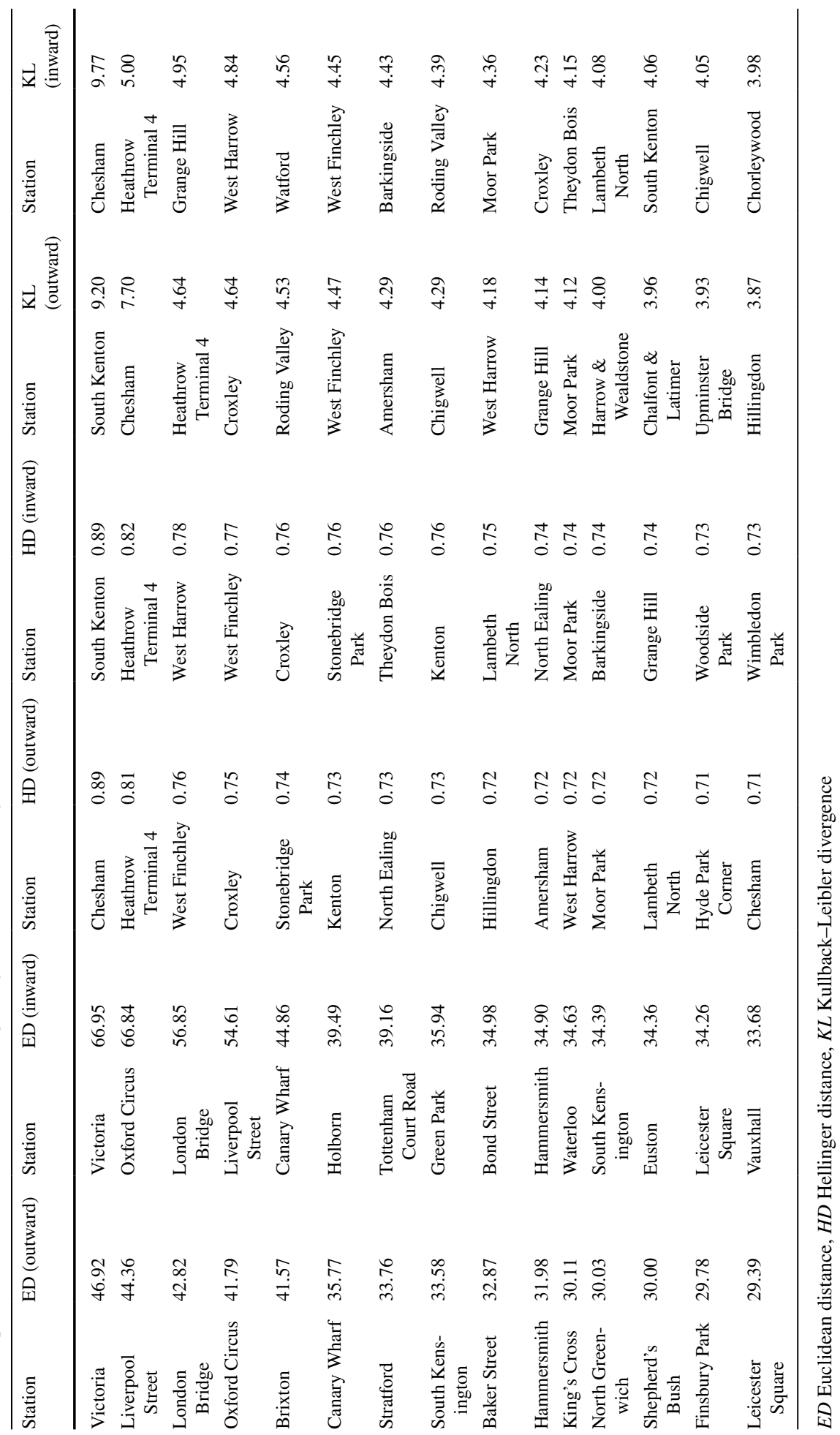




\section{Conclusions and future work}

Incidents occur frequently in urban metro systems, causing delays, crowding and substantial loss of social welfare. Operators need accurate estimates of vulnerability measures to identify the bottlenecks in the network. We propose a novel causal inference framework to estimate station-level vulnerability metrics in urban mero systems and empirically validate it for the London Underground system. In contrast to previous simulation-based studies, which largely assume virtual incident scenarios and necessitate the adoption of unrealistic assumptions on passenger behaviour, our approach relies on real incident data and avoids making behavioural assumptions by leveraging automated fare collection (smart card) data. We also illustrate that incidents can occur non-randomly, which further justifies the importance of the proposed causal inference framework in obtaining the unbiased estimate of disruption impacts.

The proposed empirical framework consists of three stages. First, we conduct propensity score matching methods and estimate unbiased disruption impacts at the station level. The estimated impacts are subsequently used to establish vulnerability metrics. In the last stage, for non-disrupted stations, we impute their vulnerability metrics by using the random forest regression model. We propose three empirical vulnerability metrics at station level, which are loss of travel demand, loss of average travel speed and loss of gross travel speed. The demand loss metrics reflects the amount of passenger who (1) switched to other transport modes, (2) switched their departure time, trip origin or destination, (3) ended their trip, before entering the disrupted metro system. In other words, it implies the demand for alternative transport services during disruptions, which can guide metro operators to prepare effective service replacement plans. The two speed related metrics reflect the degradation in the level of service for passengers who still use the metro system under disruptions. These metrics provide essential information for service recovery to mitigate the adverse influence on passengers and the overall performance of stations. The proposed irregularity in flow metrics extends the scope of vulnerability measurement to the changes in trip distribution. This irregularity metrics can be used to reflect the level of disorder within metro systems.

The results of the case study of London Underground in 2013 indicate that the effect of service disruption is heterogeneous across metro stations and it depends on the location of a station in the network and other station-level characteristics. In terms of the travel demand loss and gross speed loss (overall delay), the most affected stations are more likely to be found in Central London areas, such as Victoria, London Bridge and Liverpool Street. On the other hand, considering average speed loss (individual delay), the most affected stations are scattered around outer London areas (e.g., South Kenton and Kenton) due to lack of alternative routes.

Disruption impact estimates are probabilistic relative to the sample data, that is, causal estimates and vulnerability metrics estimates have sampling distribution. Since our analysis is based on the data of LU from October 28 to December 13, 2013, the results of our case study reflect the vulnerability status of LU for this specific period. If we use data from other periods, the estimates of vulnerability metrics might change due to inherent temporal variations in travel demand and incidents. Therefore, to improve the generalisability of vulnerability metrics estimates, the study period needs to be long enough such that the sample is representative of the population. That is, a sample should capture supply-side interruptions as much as possible, including service disruptions due to maintenance. In addition, the sample should also reflect the possible fluctuations of travel demand. 
The proposed methodology to obtain the unbiased estimates of disruption impact thus provides crucial information to metro operators for disruption management. It helps in identifying the bottlenecks in the network and in preparing targeted plans to evacuate ridership as well as to recover services in case of incidents. The direct integration of the estimated vulnerability metrics in preparing these target plans remains an avenue for future research. It is worth noting that the proposed framework can be applied to other metro systems conditional on the availability of the required data on incident logs, confounding characteristics and performance outcomes. Future empirical studies can also incorporate other context-specific and relevant confounders or outcome indicators in their analysis. For example, they can explore the disruption impacts on interchange passengers if the required datasets are available. We do not include this part of ridership in our LU case study because it cannot be directly derived from smart card data. More advanced assignment algorithm is required to identify passengers' routes by matching smart card data with vehicle location data and reproduce the spatiotemporal flow distribution in the metro network.

In line with the limitations of this study, there are three potential directions for future research. First, stations surrounding the disrupted stations may also be affected due to indirect propagation, but this study does not account for such spillover effects. Modelling spatiotemporal propagation disruption impacts requires significant methodological developments, which would be an important improvement over the current method. For instance, recent developments in Bayesian nonparametric sparse vector autoregressive models (Billio et al. 2019) can be adapted to model the spatiotemporal effect of service disruptions in transit networks. Second, the proposed vulnerability metrics can reveal static disruption impacts at different stations, but passengers need real-time service information to reschedule their trips. Thus, the current framework can be extended to update the vulnerability metrics dynamically. Considering the interaction between information provision and how it influences passengers' decision under disruptions, this advancement would improve the dissemination of the incident alerts to passengers in real-time. Finally, by merging data from other travel modes (e.g., bus, urban rails, shared bike or taxi) with metro datasets, we can estimate multi-modal vulnerability metrics in the same causal inference framework and understand the characteristics of the mode shift due to disruptions. In a potential extension of our method to multi-modal transport systems, the lost demand would not include passengers who shift to other public transport modes due to metro disruptions. Compared to metro-only vulnerability metrics, multi-modal demand loss metrics would focus on passengers who give up their trips entirely or switch to private transport modes. Therefore, for metro stations linked to multi-modal hubs, the multi-model demand loss metrics might be lower than the metro-only metrics. The magnitude of this gap would depend on the attractiveness of alternative public transport services compared to private modes.

Acknowledgements The authors are grateful for the support of Transport for London (TfL), the data provider of this research. Any opinions, findings, and conclusions or recommendations expressed in this material are those of the authors and do not necessarily reflect the views of Transport for London (TfL). We would like to thank the associate editor and three anonymous reviewers for their critical assessment of our work. Prateek Bansal was supported by the Leverhulme Trust Early Career Fellowship.

Open Access This article is licensed under a Creative Commons Attribution 4.0 International License, which permits use, sharing, adaptation, distribution and reproduction in any medium or format, as long as you give appropriate credit to the original author(s) and the source, provide a link to the Creative Commons licence, and indicate if changes were made. The images or other third party material in this article are included in the article's Creative Commons licence, unless indicated otherwise in a credit line to the material. If material is not included in the article's Creative Commons licence and your intended use is not 
permitted by statutory regulation or exceeds the permitted use, you will need to obtain permission directly from the copyright holder. To view a copy of this licence, visit http://creativecommons.org/licenses/by/4.0/.

\section{References}

Adjetey-Bahun, K., Birregah, B., Châtelet, E., Planchet, J.L.: A model to quantify the resilience of mass railway transportation systems. Reliab. Eng. Syst. Saf. 153, 1-14 (2016)

Berdica, K.: An introduction to road vulnerability: what has been done, is done and should be done. Transp. Policy 9(2), 117-127 (2002)

Billio, M., Casarin, R., Rossini, L.: Bayesian nonparametric sparse VAR models. J. Econom. 212(1), 97-115 (2019)

Brazil, W., White, A., Nogal, M., Caulfield, B., O’Connor, A., Morton, C.: Weather and rail delays: analysis of metropolitan rail in Dublin. J. Transp. Geogr. 59, 69-76 (2017)

Chopra, S.S., Dillon, T., Bilec, M.M., Khanna, V.: A network-based framework for assessing infrastructure resilience: a case study of the London metro system. J. R. Soc. Interface 13(118), 20160113 (2016)

Couronné, R., Probst, P., Boulesteix, A.L.: Random forest versus logistic regression: a large-scale benchmark experiment. BMC Bioinform. 19(1), 270 (2018)

Derrible, S., Kennedy, C.: The complexity and robustness of metro networks. Phys. A 389(17), 3678-3691 (2010)

Faturechi, R., Miller-Hooks, E.: Measuring the performance of transportation infrastructure systems in disasters: a comprehensive review. J. Infrastruct. Syst. 21(1), 04014025 (2015)

Graham, D.J., McCoy, E.J., Stephens, D.A.: Quantifying causal effects of road network capacity expansions on traffic volume and density via a mixed model propensity score estimator. J. Am. Stat. Assoc. 109(508), 1440-1449 (2014)

Hastie, T., Tibshirani, R., Friedman, J.: The Elements of Statistical Learning: Data Mining, Inference, and Prediction. Springer, Berlin (2009)

Hörcher, D., Graham, D.J., Anderson, R.J.: Crowding cost estimation with large scale smart card and vehicle location data. Transp. Res Part B: Methodol. 95, 105-125 (2017)

Imbens, G.W., Rubin, D.B.: Causal Inference in Statistics, Social, and Biomedical Sciences. Cambridge University Press, Cambridge (2015)

Imbens, G.W., Wooldridge, J.M.: Recent developments in the econometrics of program evaluation. J. Econ. Literat. 47(1), 5-86 (2009)

Jenelius, E., Petersen, T., Mattsson, L.G.: Importance and exposure in road network vulnerability analysis. Transp. Res. Part A: Policy Pract. 40(7), 537-560 (2006)

Jiang, R., Lu, Q.C., Peng, Z.R.: A station-based rail transit network vulnerability measure considering land use dependency. J. Transp. Geogr. 66, 10-18 (2018)

Khalilia, M., Chakraborty, S., Popescu, M.: Predicting disease risks from highly imbalanced data using random forest. BMC Med. Inform. Decis. Mak. 11(1), 51 (2011)

Kusakabe, T., Asakura, Y.: Behavioural data mining of transit smart card data: a data fusion approach. Transp. Res. Part C: Emerg. Technol. 46, 179-191 (2014)

Land Transport Authority: Land Transport Authority Annual Report 2016/2017 (2017). https://www.lta.gov. sg/content/dam/ltagov/who_we_are/statistics_and_publications/report/pdf/LTA_ANNUAL_REPOR T_2016-2017.pdf. Accessed 1st Mar/2020

Lechner, M.: Identification and estimation of causal effects of multiple treatments under the conditional independence assumption. In: Econometric Evaluation of Labour Market Policies, pp. 43-58. Physica, Heidelberg (2001)

Liaw, A., Wiener, M.: Classification and regression by randomForest. R News 2(3), 18-22 (2002)

Lu, Q.C.: Modeling network resilience of rail transit under operational incidents. Transp. Res. Part A Policy Pract. 117, 227-237 (2018)

Mattsson, L.G., Jenelius, E.: Vulnerability and resilience of transport systems-a discussion of recent research. Transp. Res. Part A: Policy Pract. 81, 16-34 (2015)

M'cleod, L., Vecsler, R., Shi, Y., Levitskaya, E., Kulkarni, S., Malinchik, S., Sobolevsky, S.: Vulnerability of transportation networks: the New York City Subway system under simultaneous disruptive events. Procedia Comput. Sci. 119, 42-50 (2017)

Melo, P.C., Harris, N.G., Graham, D.J., Anderson, R.J., Barron, A.: Determinants of delay incident occurrence in urban metros. Transp. Res. Rec. 2216(1), 10-18 (2011) 
Metropolitan Transportation Authority: NYC Subway Action Plan 2019. http://www.mtamovingforwar d.com/files/NYC_Subway_Action_Plan.pdf. Accessed 1st Mar. 2020

Nian, G., Chen, F., Li, Z., Zhu, Y., Sun, D.: Evaluating the alignment of new metro line considering network vulnerability with passenger ridership. Transportm. A Transp. Sci. 15(2), 1402-1418 (2019)

O’Kelly, M.E.: Network hub structure and resilience. Netw. Spatial Econ. 15(2), 235-251 (2015)

Pelletier, M.P., Trépanier, M., Morency, C.: Smart card data use in public transit: a literature review. Transp. Res. Part C: Emerg. Technol. 19(4), 557-568 (2011)

Reggiani, A., Nijkamp, P., Lanzi, D.: Transport resilience and vulnerability: the role of connectivity. Transp. Res. A: Policy Pract. 81, 4-15 (2015)

Rodríguez-Núñez, E., García-Palomares, J.C.: Measuring the vulnerability of public transport networks. J. Transp. Geogr. 35, 50-63 (2014)

Rosenbaum, P.R., Rubin, D.B.: The central role of the propensity score in observational studies for causal effects. Biometrika 70(1), 41-55 (1983)

Rubin, D.B.: Estimating causal effects of treatments in randomized and nonrandomized studies. J. Educ. Psychol. 66(5), 688 (1974)

Rubin, G.J., Brewin, C.R., Greenberg, N., Simpson, J., Wessely, S.: Psychological and behavioural reactions to the bombings in London on 7 July 2005: cross sectional survey of a representative sample of Londoners. BMJ 331(7517), 606 (2005)

Silva, R., Kang, S.M., Airoldi, E.M.: Predicting traffic volumes and estimating the effects of shocks in massive transportation systems. Proc. Natl. Acad. Sci. 112(18), 5643-5648 (2015)

Sun, D.J., Guan, S.: Measuring vulnerability of urban metro network from line operation perspective. Transp. Res. Part A: Policy Pract. 94, 348-359 (2016)

Sun, D.J., Zhao, Y., Lu, Q.C.: Vulnerability analysis of urban rail transit networks: a case study of Shanghai, China. Sustainability 7(6), 6919-6936 (2015)

Sun, H., Wu, J., Wu, L., Yan, X., Gao, Z.: Estimating the influence of common disruptions on urban rail transit networks. Transp. Res. Part A: Policy Pract. 94, 62-75 (2016)

Sun, L., Huang, Y., Chen, Y., Yao, L.: Vulnerability assessment of urban rail transit based on multi-static weighted method in Beijing, China. Transp. Res. Part A: Policy Pract. 108, 12-24 (2018)

Transport for London: London Underground Performance Report Period 1-13 2016/17 (2017). http://conte nt.tfl.gov.uk/lu-performance-report-period-13-2016-17.pdf Accessed 14th Sept. 2018

Transport for London: Underground services performance-Performance data almanac (2019). https://tfl.gov. uk/corporate/publications-and-reports/underground-services-performance. Accessed 1st Mar. 2020

UITP_International Union of Public Transport, 2018. WORLD METRO FIGURES 2018. http://www. uitp.org/sites/default/files/cck-focus-papersfiles/Statistics\%20Brief\%20-\%20World\%20metro\%20fig ures\%202018V4_WEB.pdf Accessed 1st Mar. 2020

Wan, X., Li, Q., Yuan, J., Schonfeld, P.M.: Metro passenger behaviors and their relations to metro incident involvement. Accid. Anal. Prev. 82, 90-100 (2015)

Wikimedia Commons: London Underground with Greater London map (2013). https://commons.wikim edia.org/wiki/File:London_Underground_with_Greater_London_map.svg. Accessed 19th May 2020

Willmott, C.J., Matsuura, K.: Advantages of the mean absolute error (MAE) over the root mean square error (RMSE) in assessing average model performance. Clim. Res. 30(1), 79-82 (2005)

Yang, Y., Liu, Y., Zhou, M., Li, F., Sun, C.: Robustness assessment of urban rail transit based on complex network theory: a case study of the Beijing Subway. Saf. Sci. 79, 149-162 (2015)

Yap, M., Cats, O.: Predicting disruptions and their passenger delay impacts for public transport stops. Transportation 1, 2 (2020). https://doi.org/10.1007/s11116-020-10109-9

Ye, Q., Kim, H.: Assessing network vulnerability of heavy rail systems with the impact of partial node failures. Transportation 46(5), 1591-1614 (2019)

Zhang, D.M., Du, F., Huang, H., Zhang, F., Ayyub, B.M., Beer, M.: Resiliency assessment of urban rail transit networks: Shanghai metro as an example. Saf. Sci. 106, 230-243 (2018a)

Zhang, J., Wang, S., Wang, X.: Comparison analysis on vulnerability of metro networks based on complex network. Phys. A 496, 72-78 (2018b)

Zhang, J., Xu, X., Hong, L., Wang, S., Fei, Q.: Networked analysis of the Shanghai subway network, in China. Phys. A 390(23-24), 4562-4570 (2011)

Zhang, X., Deng, Y., Li, Q., Skitmore, M., Zhou, Z.: An incident database for improving metro safety: the case of shanghai. Saf. Sci. 84, 88-96 (2016)

Zhu, S., Masud, H., Xiong, C., Yang, Z., Pan, Y., Zhang, L.: Travel behavior reactions to transit service disruptions: study of Metro SafeTrack projects in Washington, DC. Transp. Res. Rec. 2649(1), 79-88 (2017)

Zhu, Y., Goverde, R.M.: Dynamic Passenger assignment for major railway disruptions considering information interventions. Netw. Spatial Econ. 19(4), 1249-1279 (2019) 
Publisher's Note Springer Nature remains neutral with regard to jurisdictional claims in published maps and institutional affiliations.

Nan Zhang is a PhD student in the Department of Civil \& Environmental Engineering at Imperial College London. Nan received her B.Eng degree from Southeast University and MSc from Imperial College London. She is interested in causal inference methods and applications, big data analysis, and applying statisti$\mathrm{cal}$ and machine learning methods to understand disruption in urban metro systems.

Daniel J. Graham is Professor of Statistical Modelling in the Department of Civil \& Environmental Engineering at Imperial College London. Dan's research involves the development and application of statistical methods for transport analysis and modelling; causal inference methods and applications; transport and spatial economics; data centric engineering; public transport analytics; network performance and resilience; risk, safety and health.

Daniel Hörcher is Postdoctoral Research Associate at Imperial College London. He has a BSc degree from Budapest University of Technology and Economics, an MSc from VU University Amsterdam, and a PhD degree from Imperial College London. His research interests as a postdoctoral researcher are focused around the economics of public transport supply (including pricing, capacity provision, and subsidies), the wider economic benefits of public transport provision, and the statistical analysis of large-scale automated transport data.

Prateek Bansal is a Leverhulme Trust Early Career Fellow at Imperial College London (ICL). Prior to joining ICL, Prateek received his PhD degree from Cornell University (2019), MS from The University of Texas at Austin (2015), and B.Tech. from IIT Delhi (2013). He is mainly interested in creating methodological innovations at the intersection of Bayesian Machine Learning, Econometrics, and Causal Inference to address challenging questions in travel/consumer behaviour modelling and traffic safety. 